\title{
Identification of chromosomal aberrations associated with disease progression and a novel 3q13.31 deletion involving $L S A M P$ gene in osteosarcoma
}

\author{
CHUEH-CHUAN YEN ${ }^{1,2,3,10}$, WEI-MING CHEN ${ }^{4,10}$, TAIN-HSIUNG CHEN ${ }^{4,10}$, \\ WINBY YORK-KWAN CHEN ${ }^{5,14}$, PAUL CHIH-HSUEH CHEN ${ }^{5}$, HONG-JEN CHIOU ${ }^{6,10}$, \\ GIUN-YI HUNG ${ }^{7,10}$, HUNG-TA HONDAR WU ${ }^{6,10}$, CHAO-JUNG WEI ${ }^{6,10}$, CHENG-YING SHIAU ${ }^{8,10}$, \\ YU-CHUNG WU ${ }^{9,10}$, TA-CHUNG CHAO ${ }^{2,3,10}$, CHENG-HWAI TZENG ${ }^{2,3,10}$, PO-MIN CHEN ${ }^{2,3,10}$, \\ CHI-HUNG LIN ${ }^{9,11,15}$, YANN-JANG CHEN ${ }^{12,13,15}$ and JONATHAN A. FLETCHER ${ }^{1}$
}

\author{
${ }^{1}$ Department of Pathology, Brigham and Women's Hospital, 75 Francis Street, Boston, MA 02115, USA; \\ ${ }^{2}$ Genomic Research Center, ${ }^{3}$ Division of Hematology and Oncology, Department of Medicine, Departments of ${ }^{4}$ Orthopedics, \\ ${ }^{5}$ Pathology, ${ }^{6}$ Radiology, ${ }^{7}$ Pediatrics, ${ }^{8}$ Cancer Center and ${ }^{9}$ Surgery, Taipei Veterans General Hospital, No. 201, Sec. 2 , \\ Shih-Pai Road; ${ }^{10}$ School of Medicine, ${ }^{11}$ Institute of Microbiology and Immunology, ${ }^{12}$ Faculty of Life Sciences \\ and Institute of Genome Sciences and ${ }^{13}$ Institute of Clinical Medicine, National Yang-Ming University, No. 155, \\ Sec. 2, Linong Street, Taipei 112; ${ }^{14}$ Taipei Institute of Pathology, 6F, No. 146, Sec. 3, Chung-Ching N. Road; \\ ${ }^{15}$ Taipei City Hospital, No. 145, Zheng Zhou Road, Taipei 103, Taiwan, R.O.C.
}

Received January 23, 2009; Accepted March 11, 2009

DOI: 10.3892/ijo_00000390

\begin{abstract}
Five osteosarcoma (OS) cell lines, 37 OS tumors and 9 corresponding non-neoplastic samples were genotyped by Affymetrix $10 \mathrm{~K} 2.0 \mathrm{SNP}$ array. Regions of high level amplification and homozygous deletion were identified and validated by quantitative PCR and FISH. Certain recurrent cytogenetic alterations were more frequent in recurrent/ metastatic than in primary OS. These included deletion of 6q14.1, 6q16.2-q22.31, and 8p23.2-p12, amplification of $8 \mathrm{q} 21.12,8 \mathrm{q} 22.3-\mathrm{q} 24.3$ and $17 \mathrm{p} 12$, and loss of heterozygosity (LOH) at 2q24.3-q31.2, 5q11.2, 6p21.31-p21.1, 6q14.1-q16.2, 8p22-p12, 9q22.1, 10q21.1-q22.1, 10q23.31-q24.1, 12q15-q21.1 and 21q21.2-q21.3. Most of the $\mathrm{LOH}$ calls were associated with deletion, but a subset of them was associated with normal
\end{abstract}

Correspondence to: Dr Jonathan A. Fletcher, Department of Pathology, Brigham and Women's Hospital, 75 Francis Street, Boston, MA 02115, USA

E-mail: jfletcher@partners.org

Dr Chueh-Chuan Yen, Division of Hematology and Oncology, Department of Medicine, Taipei Veterans General Hospital, No. 201, Sec 2, Shih-Pai Road, Taipei 112, Taiwan, R.O.C.

E-mail: ccyen@vghtpe.gov.tw

Key words: limbic system-associated membrane protein, oligonucleotide arrays, osteosarcoma, real-time quantitative PCR, single-nucleotide polymorphism, tumor suppressor gene or increased copy number $(\mathrm{CN})$. A consensus $3 \mathrm{q} 13.31$ deletion localized to a region within the limbic systemassociated membrane protein (LSAMP) gene was also identified. The FISH evaluations demonstrated highlylocalized homozygous or heterozygous LSAMP deletions in 6 of 11 primary OS. qRT-PCR evaluations of the two major alternative $L S A M P$ transcripts demonstrated reduced expression of $1 \mathrm{~b}$ isoform transcript in each of three OS with $L S A M P$ exon $1 \mathrm{~b}$ deletion. Further, the 1a isoform transcripts in these same OS had either reduced expression or a premature termination codon in $L S A M P$ exon 2. This SNP genotyping study identified chromosomal aberrations associated with disease progression in OS and disclosed LSAMP as a novel tumor suppressor gene in OS. The study also demonstrated that $\mathrm{CN}$ and $\mathrm{LOH}$ analyses were able to detect distinct subsets of genetic abnormalities in OS.

\section{Introduction}

Previous studies have implicated several genetic mechanisms in osteosarcoma (OS) tumorigenesis. These include cell cycle alterations resulting from genomic changes of $T P 53, R B I$ and $M D M 2$; perturbation of cell senescence pathways resulting from telomere dysfunction; alterations in cell death/cytokine pathways resulting from $F A S$ dysregulation (1); and upregulation of EZR in metastatic OS (2). Genetic complexity is a hallmark of high grade OS, and multiple genomic amplifications and deletions have been demonstrated by karyotyping studies (3) and by molecular cytogenetic assays (4-10).

Previously, oligonucleotide microarrays designed for genotyping of single nucleotide polymorphisms (SNP) have 
been applied for studies of chromosomal aberrations in cancer (11-19). SNP arrays detect DNA copy number (CN) changes and cancer-specific loss of heterozygosity $(\mathrm{LOH})$ in a genomewide fashion.

In this study, we performed high-throughput SNP genotyping of 5 OS cell lines, 37 OS tumors and 9 matched non-neoplastic samples and identified chromosomal aberrations associated with disease progression in OS. A consensus 3q13.31 deletion localized to a region within the limbic system-associated membrane protein (LSAMP) gene was also identified and was associated with either reduced expression or presence of a premature termination codon of LSAMP transcripts.

\section{Materials and methods}

Primary tumors and cell lines. This study was approved by the Institutional Review Boards of Taipei Veterans General Hospital and Brigham and Women's Hospital. SNP assays were performed using genomic DNAs isolated from 37 snapfrozen OS in 36 ethnic Chinese patients (from Taipei Veterans General Hospital), matched peripheral blood lymphocytes in 9 of the patients and 5 OS cell lines. Fluorescence in situ hybridization (FISH) and quantitative reverse transcriptionPCR (qRT-PCR) were performed on an independent group of 11 frozen untreated OS from a second institution (Brigham and Women's Hospital). Informed consent was obtained from each patient. In order to minimize false-negative SNP data, the study inclusion criteria required that each OS specimen be composed of at least $70 \%$ neoplastic cells on histological review. OS cell lines (MG63, G292, U2OS, SaOS2 and HOS) and an osteoblast cell line (hFOB 1.19) were obtained from ATCC.

SNP array and dChip SNP analysis. SNP assays were performed at Microarray and Gene Expression Analysis Core Facility of the VGH-Yang Ming Genome Research Center using the GeneChip ${ }^{\circledR}$ Human Mapping 10K Array Xba 142 2.0 (GeneChip ${ }^{\circledR}$ Mapping 10K 2.0 Array) (Affymetrix, Inc., Santa Clara, CA) according to previously described methods (20) and the manufacturer's protocol. Genomic CN alterations and $\mathrm{LOH}$ changes were analyzed using dChip software (http://biosun1.harvard.edu/complab/dchip/), as previously described $(11,21,22)$. Mapping of SNP locations to cytogenetic bands was obtained by integration of datasets from Affymetrix and University of California Santa Cruz (UCSC) Genome Browser hg 17 (http://genome.ucsc.edu). All samples used in analysis had SNP call rates $>85 \%$. The invariant set normalization method (23) was used to normalize all arrays at the probe intensity level to an array with median intensity. After normalization, a model-based method was used to obtain the signal values for each SNP in each array (24).

Normalized intensities of the SNP loci obtained from 9 peripheral blood lymphocyte samples were used as the baseline to estimate the CN of the corresponding SNP loci in the tumor samples. From raw signal data, the inferred $\mathrm{CN}$ of each SNP locus was estimated by applying the hidden Markov model (HMM) (11). An inferred copy step of 0.335 was used as modified from Zhao et al (19), based on the possibility of diploidy or triploidy in the cancer cells.
The SNP calls of a matched pair of peripheral blood lymphocyte and tumor samples were combined to obtain the observed LOH calls. For unpaired tumor samples or cell lines, regions with $\mathrm{LOH}$ call were identified using an HMM method developed by Beroukhim et al (22). Regions of $\mathrm{CN}$-neutral $\mathrm{LOH}$ were identified by setting $\mathrm{LOH}$ with $\mathrm{CN}$ $2 \pm 0.335$.

Quantitative PCR ( $q P C R$ ) confirmation of SNP findings. All primers were designed with Primer Express 3.0 software (Applied Biosystems Foster City, CA) using default parameters, with modified minimum amplicon length requirements (85 bp) and with requirement of maximum GC content of $40 \%$ for the five last $3^{\prime}$ end nucleotides. The primer sequences are shown in Table I. PCR reactions were performed, as previously described (25). DNA content was normalized to that of long interspersed elements (LINE1), a repetitive element for which CNs per haploid genome are similar both in normal or neoplastic cells (26). $\mathrm{CN}$ changes per haploid genome were calculated using the formula $2 * 2^{\text {(Nt-Nline)-(Tt-Tline), }}$ where Nt and Nline are the threshold cycle numbers observed for the experimental primer and LINE1 primer, respectively, in the normal DNA samples, and Tt and Tline are the threshold cycle numbers observed for the experimental primer and LINE1 primer, respectively, in the test DNA samples (26). In normal cells the expected $\mathrm{CN}$ of each gene is 2 . PCR reactions with each primer set were performed in triplicate, and calculated $\mathrm{CN}$ changes per haploid genome were averaged.

FISH. FISH was performed using methods described previously $(27,28)$. FISH probes were made from bacterial artificial chromosome (BAC) clones - containing genomic segments of interest - identified using Ensembl Genome Browser http://www.ensembl.org/ and UCSC Genome Browser http://genome.ucsc.edu. BACs were obtained from the RPCI-11 BAC library or CTD clone collection $(29,30)$, digoxigenin-labeled using the Random Prime DNA Labeling System (Invitrogen) according to the manufacturer's protocol and detected with FITC (green). Biotin-labeled probe to the chromosome 3 centromeric region was purchased from Zymed/ Invitrogen and was detected with rhodamine (red). BAC chromosomal locations were corroborated by FISH in normal lymphocyte metaphases. OS FISH assays were scored only in neoplastic nuclei, and the relative $\mathrm{CN}$ per nucleus was defined as (\#BAC signals/\#centromeric signals) x 2. Samples where $>50 \%$ of nuclei had relative FISH CN of $\leq 1$ or 0 , respectively, were considered to have heterozygous or homozygous deletions.

$R N A$ preparation, $q R T-P C R, R T-P C R$ and sequencing. Total RNAs were isolated from cell lines and frozen tumors using TRIzol ${ }^{\circledR}$ Reagent (Invitrogen), according to the manufacturer's instructions. Reverse transcription was performed from $1 \mu \mathrm{g}$ RNA using the Bio-Rad iScript ${ }^{\mathrm{TM}}$ cDNA synthesis kit (Bio-Rad Laboratories, Hercules, CA). mRNA CN for both $L S A M P$ isoforms and for the $\beta$-actin housekeeping gene were determined by qRT-PCR using iQ SYBR-Green supermix and a MyiQ single-color real-time PCR detection system (Bio-Rad Laboratories). Primers to amplify type 1a and type $1 \mathrm{~b}$ 
Table I. Primer sequences for genomic quantitative PCR.

\begin{tabular}{|c|c|c|}
\hline Gene & Forward & Reverse \\
\hline PDGFRA & TCTTCTGCCTCCCACTCCAT & TGTTTGACAGTGTGGATAGATGTGA \\
\hline DNAH8 & GAAGAAGCTATTCCTGCGAGGAA & TCACATCGCGGTCCTCTGT \\
\hline$D A A M 2$ & TGGTAGCCCAGCTCAGTGAA & ACAGCCTCAAGGGTCTCTTCTATC \\
\hline$C D C 5 L$ & CAATGCAGCTAGAGTGTCAAGAATG & AGGAGATAGTGATGTTTCTAAAACCAAA \\
\hline SDK1 & CAGGTGGCAACACATGCTTT & GAGGTCTACTGAACACTGCCAGAA \\
\hline SLC $30 A 8$ & AGCCAAGTGGTTCGGAGAGA & ATGTTTGAAGGTGGCCTGTCA \\
\hline$R A B 38$ & TTTAATCAGCAAAGGCCTCAAGT & GCCGAGAATGGAGGTCATCTT \\
\hline LRIG3 & TCTGAGCTAGTTCTGTGTGGGATT & AGATCAAAGGGTACCGCATGTC \\
\hline MDM2 & GGGAAGAAACCCAAGACAAAGA & GGGCAGGGCTTATTCCTTTT \\
\hline COPS3 & ATGGGCTCACAAGAAGATGATTC & CTGCCCTCCGAACACTTGTC \\
\hline BTG3 & CTTCCAATGTGGCACCCTTT & TGATTCCGGTCACAATGCAT \\
\hline$L S A M P$ & TCGCAGCGTGGATTTTAACC & GAGCTGGAGTTCAAGGAGATCAG \\
\hline CNTNAP2 & TTGGGACAAAAATAACGAGCAA & TCCCTCTTTATCGTGGATTCCA \\
\hline$C D K N 2 A$ & TGGAGAGGTGACCCAGAGTTG & CCGCGCGCACTTTCC \\
\hline LOC 389707 & TGAGGCAGGAAGGTTTCAGTTC & TGTCAGCTAGGAGGACTTCCAAT \\
\hline LINE-1 & CCGCTCAACTACATGGAAACTG & GCGTCCCAGAGATTCTGGTATG \\
\hline
\end{tabular}

LSAMP isoforms were designed based on full length LSAMP 1b isoform mRNA (Genbank accession number NM_002338) and cDNA sequence BI199955 covering exon 1a (31), so that both primer pairs shared reverse primer within exon 4 (5'-AAATTCCCTTCCAGTTGGTGTAA GGT-3') and forward primers within exons 1a (5'-GACCTA CTGGCTGCACAGCGTCT-3') or 1b (5'-GTTCAGCCGGA TCGGAAACAGT-3'). ß-actin (forward primer 5'-CACTGC CCTGGCACCCAGCACA-3' and reverse primer 5'-AGAG GACTGGGCCATTCTCCTT-3') levels were used to normalize the investigated samples, and hFOB 1.19 was used as baseline comparator (32). Cycling conditions were as follows: $95^{\circ} \mathrm{C}$ for $3 \mathrm{~min}$ and then 50 cycles at $95^{\circ} \mathrm{C}$ for $10 \mathrm{sec}$, $62^{\circ} \mathrm{C}$ for $30 \mathrm{sec}$ and $68^{\circ} \mathrm{C}$ for $1 \mathrm{~min}$. Gene expression levels were calculated, as described previously (33). For RT-PCR and sequencing of $1 \mathrm{a}$ isoforms, forward primer 5'-AGGACC TACTGGCTGCACAGCGTC-3' and reverse primer 5'-CCC TCATCATAGACATCCACCTTCTGGA-3' were used. Cycling conditions were as follows: $95^{\circ} \mathrm{C}$ for $5 \mathrm{~min}$ and then 50 cycles at $95^{\circ} \mathrm{C}$ for $15 \mathrm{sec}, 65^{\circ} \mathrm{C}$ for $30 \mathrm{sec}$ and $68^{\circ} \mathrm{C}$ for $1 \mathrm{~min}$.

Statistical analysis. Statistical comparisons were performed using SPSS 12.0 software. Chi-square tests or Fisher exact tests were used to evaluate the correlations between chromosomal aberrations and tumor status (primary vs. recurrent/ metastatic). A P-value $<0.05$ was considered statistically significant.

\section{Results}

Clinicopathological characteristics. Clinicopathological characteristics of the 36 patients (23 males and 13 females) are summarized in Table II. For Case OS51, both a recurrent OS and a lung metastasis were analyzed from the same patient. The 37 tumor samples included 23 primary bone tumors, 7 locally recurrent bone tumors and 7 metastases. The histological subtypes included 23 osteoblastic OS, 7 chondroblastic OS, 5 fibroblastic OS, 1 telangiectatic OS and 1 osteoblastoma-like OS.

SNP evaluations of chromosomal aberrations in 42 samples. SNP genome-wide evaluations of $\mathrm{CN}$ change are summarized in Fig. 1. Frequent chromosomal regions of $\mathrm{CN}$ gain (regions $>5 \mathrm{~kb}$ containing at least three consecutive SNPs with average inferred $\mathrm{CN} \geq 3$ occurring in 6 or more cases) and $\mathrm{CN}$ loss (regions $>5 \mathrm{~kb}$ containing at least three consecutive SNPs with average inferred $\mathrm{CN} \leq 1.5$ occurring in 6 or more cases) are summarized in Table III. The size of regions of frequent aberrations ranged from $<0.91$ to $>44.08 \mathrm{Mb}$, the number of SNP spanning these regions ranged from 4 to 186 and the number of gene contained with these regions ranged from 3 to 246 . Most frequent ( $>20 \%$ ) $\mathrm{CN}$ gain was found over 8q22.2-q24.22 and 17p, and most frequent CN loss was identified at 2q33.2-q33.3, 2q36.3-q37.3, 3p21.2 and $3 q 13.31$.

High-level amplifications, defined as regions $>5 \mathrm{~kb}$ containing at least three consecutive SNPs with average inferred $\mathrm{CN} \geq 6$ in two or more specimens, were identified at chromosome bands 4q12, 6p21.2-p21.1, 7p22.2, 8q24.11, $11 \mathrm{q} 14.2,12 \mathrm{q} 14.1,12 \mathrm{q} 15,17 \mathrm{p} 12$ and 21q21.1. Homozygous deletions, defined as regions $>5 \mathrm{~kb}$ containing at least three consecutive SNPs with average inferred $\mathrm{CN} \leq 0.67$ in two or more cases, with at least one case having an inferred $\mathrm{CN}$ $\leq 0.34$, were identified at chromosome bands $3 q 13.31,7 q 35$ and $9 \mathrm{p} 21.3$. $\mathrm{CN}$ alterations for genes in these regions were verified by qPCR (Table IV), and were consistent with the SNP dChip findings, although gene amplifications were saturated at a lower level when appraised by dChip compared to qPCR. Thus, dChip inferred $\mathrm{CN}$ of $\geq 6$ was found to represent actual (qPCR) locus $\mathrm{CN}$ ranging from 6.17 to 40 (Table IV).

The LOH analyses are summarized in Fig. 1. Chromosomal regions associated with frequent $\mathrm{LOH}$ ( $>30 \%$ of cases) 
Table II. Clinicopathological characteristics of 36 OS patients.

\begin{tabular}{|c|c|c|c|c|c|}
\hline Case no. & Age & Gender & Histology & Tumor site & Tumor nature \\
\hline OS1 & 16 & $\mathrm{~F}$ & $\mathrm{CHO}$ & Lung & Metastasis \\
\hline OS20 & 15 & $\mathrm{~F}$ & OST & Proximal fibula & Recurrent \\
\hline OS21 & 42 & M & FIB & Lung & Metastasis \\
\hline OS31 & 18 & $\mathrm{~F}$ & OST & Lung & Metastasis \\
\hline OS32 & 17 & M & OST & Proximal humerus & Primary \\
\hline OS33 & 67 & M & FIB & Proximal humerus & Primary \\
\hline OS35 & 21 & M & OST & Distal femur & Primary \\
\hline OS36 & 55 & $\mathrm{~F}$ & FIB & Proximal tibia & Primary \\
\hline OS37 & 15 & M & OST & Distal femur & Primary \\
\hline OS39 & 18 & M & FIB & Distal femur & Primary \\
\hline OS40 & 11 & $\mathrm{~F}$ & OST & Distal femur & Primary \\
\hline OS41 & 15 & M & OST & Proximal tibia & Primary \\
\hline OS42 & 19 & M & OST & Lung & Metastasis \\
\hline OS43 & 6 & $\mathrm{~F}$ & OST & Proximal tibia & Primary \\
\hline OS46 & 25 & M & OST & Proximal tibia & Primary \\
\hline OS47 & 15 & M & OST & Distal femur & Primary \\
\hline OS51T & 18 & M & $\mathrm{CHO}$ & Distal femur & Recurrent \\
\hline OS51L $\mathrm{L}^{\mathrm{a}}$ & 18 & M & $\mathrm{CHO}$ & Lung & Metastasis \\
\hline OS55-R & 39 & $\mathrm{~F}$ & $\mathrm{CHO}$ & Proximal humerus & Primary \\
\hline OS56 & 14 & $\mathrm{~F}$ & OST & Lung & Metastasis \\
\hline $\mathrm{O} 3$ & 71 & $\mathrm{~F}$ & OST & Distal femur & Primary \\
\hline $12 \mathrm{~T}$ & 13 & M & $\mathrm{CHO}$ & Distal femur & Primary \\
\hline $13 \mathrm{~T}$ & 15 & M & OST & Distal femur & Primary \\
\hline $14 \mathrm{~T}$ & 11 & M & TEL & Proximal humerus & Primary \\
\hline $15 \mathrm{~T}-\mathrm{R}$ & 13 & M & OST & Distal femur & Primary \\
\hline $17 \mathrm{~T}$ & 30 & $\mathrm{~F}$ & FIB & Proximal tibia & Recurrent \\
\hline $18 \mathrm{~T}$ & 18 & M & OST & Distal femur & Recurrent \\
\hline $22 \mathrm{~T}$ & 12 & M & $\mathrm{CHO}$ & Proximal humerus & Recurrent \\
\hline $24 \mathrm{~T}$ & 16 & $\mathrm{~F}$ & OST & Distal femur & Primary \\
\hline $25 \mathrm{~T}$ & 16 & M & OST & Distal femur & Primary \\
\hline $26 \mathrm{~T}$ & 49 & $\mathrm{~F}$ & OSTBA & External ear canal & Metastasis \\
\hline $31 \mathrm{~T}$ & 15 & $\mathrm{~F}$ & OST & Proximal tibia & Primary \\
\hline $32 \mathrm{~T}$ & 25 & M & $\mathrm{CHO}$ & Proximal tibia & Primary \\
\hline $33 \mathrm{~T}$ & 16 & M & OST & Distal femur & Primary \\
\hline $34 \mathrm{~T}$ & 6 & M & OST & Proximal humerus & Recurrent \\
\hline $35 \mathrm{~T}$ & 21 & M & OST & Distal femur & Primary \\
\hline $36 \mathrm{~T}$ & 7 & $\mathrm{~F}$ & OST & Distal femur & Recurrent \\
\hline
\end{tabular}

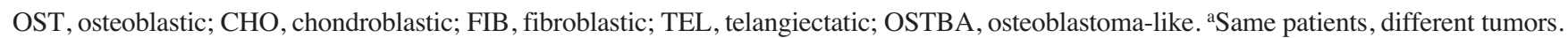

included 3p, 6q, 8p, 9p, 10q and 13q. Although most of these LOH events corresponded to physical deletions, $28 \%$ of $\mathrm{LOH}$ events appeared to result from $\mathrm{CN}$-neutral $\mathrm{LOH}$. CN-neutral $\mathrm{LOH}$ regions were frequently seen $(>60 \%$ of $\mathrm{LOH}$ were CN-neutral LOH) at 1p, 1q, 2p23-p16, 2q32.3-q33.1, 3q29, 4q13.3, 7q31.1-q31.2, 9q32-q33, 11p11.2-p11.12, 11q, 12p12.1-p11.21, 14q21, 15q, 16q23.1-q24.3 and 20q12.

Identifications of chromosomal aberrations associated with recurrent/metastatic OS. As shown in Fig. 2 and Table V, recurrent/metastatic tumors were associated with $\mathrm{CN}$ gain over 8q21.12 (79.74-91.94 Mb), 8q22.3-q24.3 (104.79$142.07 \mathrm{Mb})$ and $17 \mathrm{p} 12(14.69-17.35 \mathrm{Mb})$. On the other hand, gains involving Xp11.22 (54.34-56.77 Mb) was more often seen in primary than recurrent/metastatic tumors.
Deletions more frequently found in recurrent/metastatic tumors included 6q14.1 (83.02-85.96 Mb), 6q16.2-q22.31 (99.36-121.40 Mb) and 8p23.2-p12 (5.26-32.65 Mb). On the other hand, deletion involving 13q31.3 (92.90-114.04 Mb) was more often seen in primary than recurrent/metastatic tumors (Fig. 2 and Table V).

Regions with LOH calls significantly associated with recurrent/metastatic OS included 2q24.3-q31.2 (164.56178.77 Mb), 5q11.2 (54.87-57.11 Mb), 6p21.31-p21.1 (34.59-46.15 Mb), 6q14.1-q16.2 (79.99-99.36 Mb), 8p22-p12 (13.06-32.65 Mb), 9q22.1 (88.54-97.56 Mb), 10q21.1-q22.1 (57.90-72.38 Mb), 10q23.31-q24.1 (90.41-97.48 Mb), 12q15q21.1 (69.68-72.26 Mb) and 21q21.2-q21.3 (24.36-28.29 Mb) (Table VI). Among these, the 6q, 8p, and 10q LOH events generally resulted from $\mathrm{CN}$ losses (deletions). However, a 


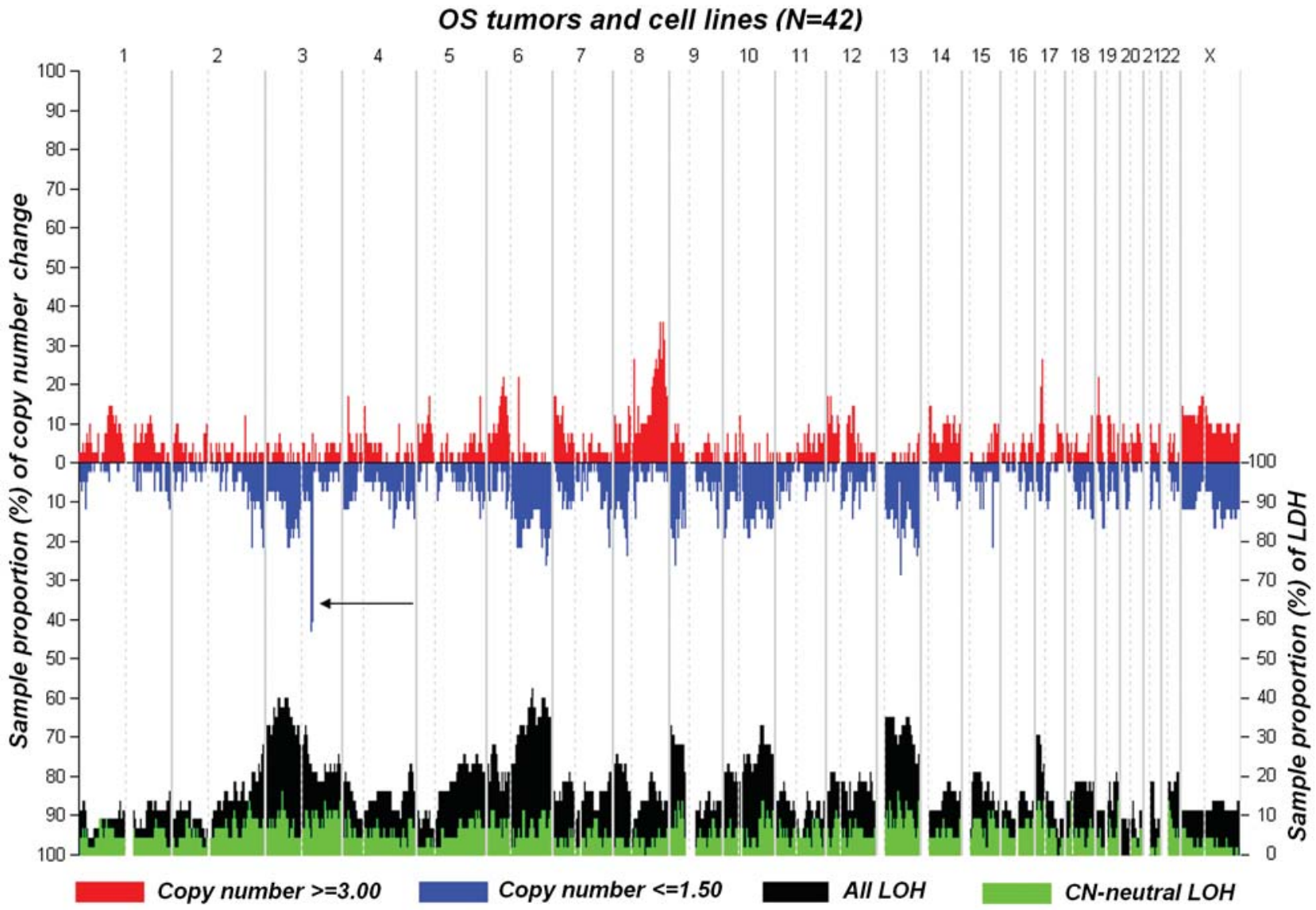

Figure 1. Summary plot of percentage of genome-wide inferred copy number (CN) alterations (red, percentage of $\mathrm{CN} \geq 3$; blue, percentage of $\mathrm{CN} \leq 1.5)$ as well as LOH (overall, black; CN-neutral LOH, green) in the OS samples and cell lines. A highly-localized deletion site is identified at 3q13.31 (arrow).

\section{A Primary tumors $(\mathrm{N}=23)$}
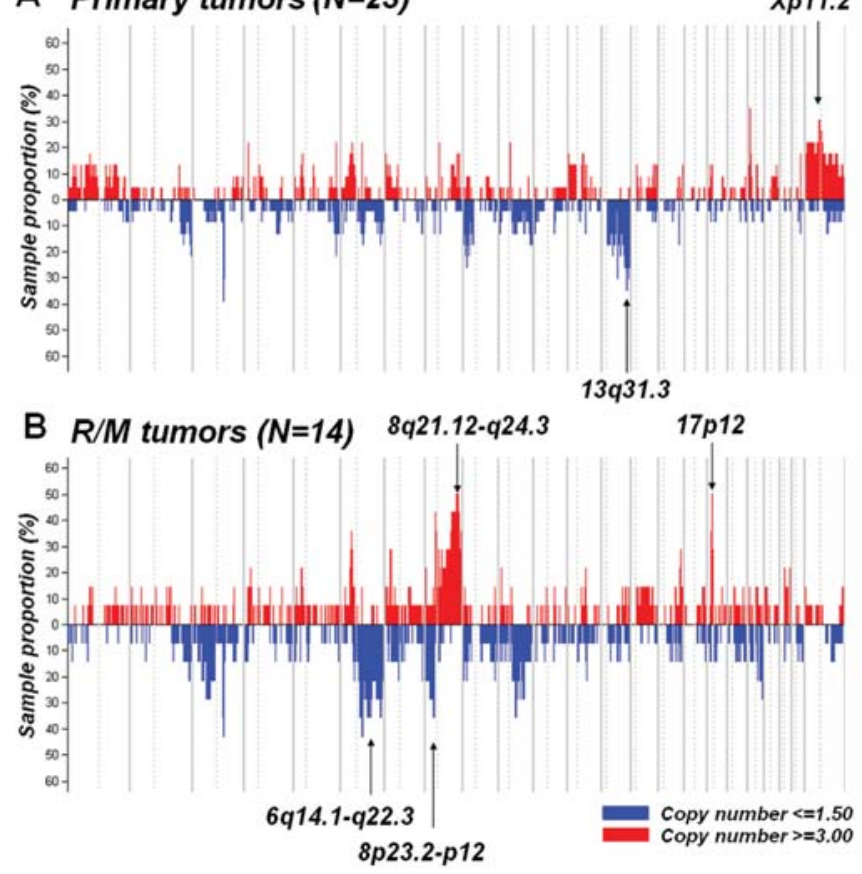

Figure 2. (A) Chromosomal aberration profiles in primary vs. (B) recurrent/metastatic $(\mathrm{R} / \mathrm{M})$ OS. Deletion of $6 \mathrm{q} 14.1-\mathrm{q} 22.31$ and $8 \mathrm{p} 23.2-\mathrm{p} 12$ as well as amplification of $8 \mathrm{q} 21.12-\mathrm{q} 24.3$ and $17 \mathrm{p} 12$ were more frequent in recurrent/metastatic than in primary tumors. Primary tumors had a higher frequency of Xp11.22 gains and 13q31.3 deletion. See Table V for details. subset of $\mathrm{LOH}$ events was CN-neutral, such as the $2 \mathrm{q} \mathrm{LOH}$ in case $34 \mathrm{~T}$ (Fig. 3 and Table VI). Some regions with LOH call were associated with increased CN (mono-allelic amplifications), including those at 6p21.2-p12.1 (39.87$53.54 \mathrm{Mb})$ in case $22 \mathrm{~T}, 12 \mathrm{q} 15(66.97-70.32 \mathrm{Mb})$ in case $26 \mathrm{~T}$, and 21q21.1-q22.11 (15.83-33.41 Mb) in case OS51T (Fig. 3 and Table VI). CN changes in these three cases were confirmed by qPCR (Table IV).

Evaluation of the novel deletion region 3q13.31. A highlylocalized region extending from 117.39 to $118.54 \mathrm{Mb}$ at 3 q13.31 was identified as a novel deletion region by SNP analysis (Figs. 1 and 4). This region of deletion is within the LSAMP gene, which is the only known gene in this region. LSAMP is transcribed in a centromeric direction and two known human isoforms are resulted from alternative use of exon 1b (genomic LSAMP isoform 1b spanning 117.01$117.64 \mathrm{Mb}$ ) or exon 1a (genomic LSAMP isoform 1a spanning 117.01-119.20 Mb) (31) (Fig. 4D). Three cell lines, G292, MG63 and HOS, had highly-localized LSAMP deletions by SNP array (Table IV and Fig. 4A-C). These deletions were corroborated by FISH using bacterial artificial chromosome (BAC) clones RP11-33E19 (117.46-117.61 Mb, containing LSAMP exon 1b), and CTD-2119D22 (118.05-118.13 Mb, within a large intron between $L S A M P$ exons 1a and 1b). HOS cells had homozygous deletion of both BACs. MG63 and G292 cells had homozygous and heterozygous CTD-2119D22 
Table III. Regions of frequent copy number gain or loss.

\begin{tabular}{|c|c|c|c|c|c|c|c|}
\hline Chromosome band & Start (Mb) & Stop (Mb) & Size (Mb) & SNP no. & Gain/loss & Rate $(\%)$ & Gene no. ${ }^{a}$ \\
\hline $2 q 33.2-q 33.3$ & 204.25 & 208.00 & 3.75 & 19 & Loss & 21.43 & 23 \\
\hline $2 q 36.3-q 37.3$ & 230.54 & 240.64 & 10.10 & 24 & Loss & 23.81 & 131 \\
\hline $3 \mathrm{p} 22.3$ & 35.96 & 39.85 & 3.89 & 16 & Loss & 14.29 & 46 \\
\hline $3 \mathrm{p} 21.2$ & 52.86 & 85.90 & 33.04 & 126 & Loss & 21.43 & 134 \\
\hline $3 q 13.31$ & 117.63 & 118.54 & 0.91 & 11 & Loss & 45.24 & 4 \\
\hline $4 q 12$ & 53.72 & 55.55 & 1.83 & 13 & Gain & 14.29 & 12 \\
\hline $4 q 35.2$ & 187.77 & 191.09 & 3.33 & 23 & Loss & 16.67 & 20 \\
\hline $5 \mathrm{p} 13.3$ & 31.05 & 33.20 & 2.15 & 9 & Gain & 14.29 & 17 \\
\hline $5 q 34$ & 162.93 & 165.27 & 2.34 & 10 & Loss & 16.67 & 3 \\
\hline $6 \mathrm{p} 21.2-\mathrm{p} 21.1$ & 38.77 & 45.61 & 6.84 & 26 & Gain & 19.05 & 105 \\
\hline $6 \mathrm{q} 14.1-\mathrm{q} 22.31$ & 76.78 & 120.86 & 44.08 & 186 & Loss & 19.05 & 246 \\
\hline $6 \mathrm{q} 23.3-\mathrm{q} 27$ & 137.22 & 170.51 & 33.29 & 146 & Loss & 19.05 & 232 \\
\hline $7 \mathrm{p} 22.3$ & 1.84 & 7.26 & 5.43 & 14 & Gain & 19.05 & 68 \\
\hline $7 q 35-q 36.3$ & 143.95 & 155.13 & 11.17 & 38 & Loss & 14.29 & 104 \\
\hline 8p21.2-p12 & 24.68 & 36.84 & 12.16 & 58 & Loss & 16.67 & 76 \\
\hline $8 \mathrm{q} 22.2-\mathrm{q} 24.22$ & 101.56 & 142.07 & 40.51 & 149 & Gain & 33.33 & 168 \\
\hline $9 \mathrm{p} 24.3-\mathrm{p} 21.3$ & 0.24 & 25.25 & 25.01 & 165 & Loss & 19.05 & 136 \\
\hline 10p15.3-p14 & 1.37 & 10.95 & 9.59 & 48 & Loss & 14.29 & 54 \\
\hline $10 \mathrm{q} 21.1-\mathrm{q} 21.3$ & 52.92 & 67.65 & 14.73 & 77 & Loss & 19.05 & 46 \\
\hline $10 \mathrm{q} 24.1-10 \mathrm{q} 24.2$ & 97.06 & 101.75 & 4.69 & 33 & Loss & 16.67 & 61 \\
\hline $10 \mathrm{q} 25.3-\mathrm{q} 26.3$ & 118.59 & 133.79 & 15.20 & 58 & Loss & 19.05 & 118 \\
\hline $12 \mathrm{p} 13.31-\mathrm{p} 13.32$ & 9.29 & 10.70 & 1.41 & 6 & Gain & 16.67 & 40 \\
\hline $12 q 15$ & 66.79 & 68.40 & 1.62 & 13 & Gain & 14.29 & 21 \\
\hline $13 q 12.2-q 12.3$ & 27.78 & 30.91 & 3.14 & 25 & Loss & 14.29 & 23 \\
\hline $13 q 14.11-q 14.3$ & 41.69 & 52.31 & 10.62 & 48 & Loss & 14.29 & 109 \\
\hline $13 q 21.1-q 21.33$ & 58.26 & 72.09 & 13.84 & 61 & Loss & 19.05 & 25 \\
\hline 13q31.2-q34 & 87.00 & 114.04 & 27.04 & 128 & Loss & 16.67 & 138 \\
\hline $17 \mathrm{p} 12$ & 14.30 & 17.35 & 3.05 & 12 & Gain & 26.19 & 52 \\
\hline $18 \mathrm{q} 22.3-\mathrm{q} 23$ & 68.37 & 72.21 & 3.84 & 15 & Loss & 14.29 & 16 \\
\hline $19 \mathrm{p} 12$ & 20.15 & 22.01 & 1.86 & 5 & Loss & 14.29 & 21 \\
\hline $19 q 13.43$ & 61.13 & 63.44 & 2.31 & 6 & Loss & 16.67 & 77 \\
\hline $\mathrm{Xp} 11.22$ & 54.34 & 56.77 & 2.43 & 4 & Gain & 14.29 & 26 \\
\hline
\end{tabular}

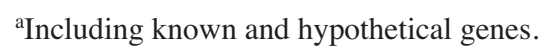

deletions, respectively, but lacked RP11-33E19 deletion (Fig. 5).

Confirmation of localized LSAMP deletion in primary OSs and correlation of expression with deletion. FISH analyses demonstrated localized LSAMP deletions in 6 of 11 primary OS. Four OS (ST90-0169, ST89-0145, ST92-307 and ST01-314) had homozygous or heterozygous CTD-2119D22 deletion only. Two OS (ST98-0626 and ST98-0878) had both CTD-2119D22 and RP11-33E19 deletion. The deletion regions of ST98-0878 also involved RP11-1033G6 (119.07-119.24 Mb, covering $L S A M P$ exon 1a) (Fig. 6A).

The genes for $L S A M P$ isoforms $1 \mathrm{a}$ and $1 \mathrm{~b}$ differ only in their signaling peptides (31). Therefore we implemented a qRT-PCR strategy using a single exon 4 reverse primer with exon 1a or 1b-specific forward primers to detect the alternative LSAMP transcripts (Fig. 6A). The primary OS (ST98-0878 and ST98-0626), as described above, with heterozygous LSAMP FISH deletions affecting exon $1 \mathrm{~b}$, and the HOS cell line with homozygous deletion of exon $1 \mathrm{~b}$, all had expected low expression of LSAMP $1 \mathrm{~b}$ (Fig. 6B). In addition, ST980878 also had low LSAMP 1a expression, most likely explained by the large heterozygous deletion (extending from 117.46 to $119.24 \mathrm{Mb}$ ) involving LSAMP exon 1a in that case. However, ST98-0626 and HOS, which had deletions limited to the 117.46 to $118.13 \mathrm{Mb}$ regions, had high LSAMP 1a expression (Fig. 6C). Representative figures of FISH results are shown in Fig. 7.

Demonstration of a truncated LSAMP 1a transcript with premature stop codon in HOS and ST98-0626. RT-PCR analyses of $L S A M P$ transcript 1a, performed using primers in exon 1a and exon 2, revealed a 308 bp expressed sequence in hFOB 1.19, MG63 and G292, whereas HOS cells expressed a 229 bp sequence, and ST98-0626 expressed both the 229 and 308 bp sequences (Fig. 8A). Sequencing analyses showed that the $308 \mathrm{bp}$ fragment resulted from the splicing of exon 1a to the 75 th bp of exon $1 \mathrm{~b}$, which was in turn spliced to exon 2 . 
Table IV. Regions of high amplification and homozygous deletion detected by SNP arrays and qPCR of candidate genes.

\begin{tabular}{|c|c|c|c|c|c|c|c|c|c|}
\hline Cytoband & $\begin{array}{l}\text { Start } \\
(\mathrm{Mb})\end{array}$ & $\begin{array}{l}\text { Stop } \\
(\mathrm{Mb})\end{array}$ & $\begin{array}{l}\text { Size } \\
(\mathrm{Mb})\end{array}$ & SNP no. & Sample & $\begin{array}{l}\text { Mean dChip } \\
\text { copy no. }\end{array}$ & Gene no. & $\begin{array}{l}\text { Candidate } \\
\text { genes }\end{array}$ & $\begin{array}{c}\text { qPCR of } \\
\text { candidate genes }\end{array}$ \\
\hline \multirow[t]{3}{*}{$4 q 12$} & 53.72 & 56.45 & 2.73 & 9 & $14 \mathrm{~T}$ & 8.00 & \multirow[t]{3}{*}{19} & \multirow[t]{3}{*}{ PDGFRA } & 8.94 \\
\hline & 53.72 & 56.45 & 2.73 & 9 & $25 \mathrm{~T}$ & 8.00 & & & 8.85 \\
\hline & 53.72 & 55.55 & 1.83 & 7 & $32 \mathrm{~T}$ & 8.00 & & & 11.52 \\
\hline \multirow[t]{2}{*}{$6 \mathrm{p} 21.2-6 \mathrm{p} 21.1$} & 38.77 & 39.06 & 0.29 & 4 & OS56 & 8.00 & \multirow[t]{2}{*}{2} & \multirow[t]{2}{*}{ DNAH8 } & 13.29 \\
\hline & 38.77 & 39.06 & 0.29 & 4 & MG63 & 6.00 & & & 9.04 \\
\hline \multirow[t]{2}{*}{$6 \mathrm{p} 21.2-6 \mathrm{p} 21.1$} & 39.87 & 41.31 & 1.44 & 10 & OS56 & 6.60 & \multirow[t]{2}{*}{19} & \multirow[t]{2}{*}{$D A A M 2$} & 10.85 \\
\hline & 39.87 & 41.31 & 1.44 & 10 & $22 \mathrm{~T}$ & 6.00 & & & 13.35 \\
\hline \multirow[t]{2}{*}{$6 \mathrm{p} 21.1$} & 44.35 & 44.87 & 0.52 & 8 & $22 \mathrm{~T}$ & 6.00 & \multirow[t]{2}{*}{5} & \multirow[t]{2}{*}{$C D C 5 L$} & 11.18 \\
\hline & 44.35 & 44.87 & 0.52 & 8 & $34 \mathrm{~T}$ & 6.00 & & & 10.99 \\
\hline \multirow[t]{2}{*}{$7 \mathrm{p} 22.2$} & 3.27 & 3.30 & 0.03 & 3 & OS43 & 10.00 & \multirow[t]{2}{*}{1} & \multirow[t]{2}{*}{$S D K 1$} & 9.75 \\
\hline & 3.27 & 3.30 & 0.03 & 3 & U2OS & 6.00 & & & 6.17 \\
\hline \multirow[t]{2}{*}{$8 q 24.11$} & 116.76 & 118.47 & 1.71 & 11 & OS47 & 6.00 & \multirow[t]{2}{*}{6} & \multirow[t]{2}{*}{ SLC $30 A 8$} & 9.89 \\
\hline & 116.76 & 118.47 & 1.71 & 11 & MG63 & 6.00 & & & 20.34 \\
\hline \multirow[t]{2}{*}{$11 \mathrm{q} 14.2$} & 86.43 & 87.65 & 1.22 & 9 & OS31 & 6.00 & \multirow[t]{2}{*}{4} & $R A B 38$ & 11.01 \\
\hline & 86.43 & 87.65 & 1.22 & 9 & G292 & 6.00 & & & 15.62 \\
\hline $12 q 14.1$ & 56.68 & 57.76 & 1.08 & 10 & OS43 & 6.00 & 3 & LRIG3 & 40.71 \\
\hline & 56.68 & 57.76 & 1.08 & 10 & $26 \mathrm{~T}$ & 8.00 & & & 26.90 \\
\hline $12 q 15$ & 66.97 & 67.67 & 0.70 & 3 & OS55-R & 6.00 & 10 & $M D M 2$ & 10.21 \\
\hline & 66.97 & 67.67 & 0.70 & 3 & $26 \mathrm{~T}$ & 8.00 & & & 20.86 \\
\hline $17 \mathrm{p} 12$ & 15.11 & 17.35 & 2.24 & 5 & OS51T & 8.00 & 50 & COPS3 & 9.72 \\
\hline & 15.11 & 17.35 & 2.24 & 5 & OS51L & 8.00 & & & 9.15 \\
\hline & 15.11 & 17.35 & 2.24 & 5 & G292 & 6.00 & & & 7.55 \\
\hline $21 \mathrm{q} 21.1$ & 17.03 & 20.51 & 3.48 & 20 & OS51T & 8.00 & 13 & BTG3 & 9.32 \\
\hline & 17.03 & 20.51 & 3.48 & 20 & OS51L & 6.00 & & & 8.16 \\
\hline $3 q 13.31$ & 117.57 & 117.75 & 0.18 & 5 & OS40 & 0.34 & 1 & $L S A M P, 1 a$ & \\
\hline & & & & & & & & $\& 1 b$ & 0.15 \\
\hline & 117.63 & 117.75 & 0.12 & 3 & $22 \mathrm{~T}$ & 0.67 & & & 0.55 \\
\hline & 117.63 & 117.75 & 0.12 & 3 & $33 \mathrm{~T}$ & 0.34 & & & 0.14 \\
\hline & 117.39 & 117.75 & 0.36 & 6 & HOS & 0.00 & & & 0.00 \\
\hline $3 q 13.31$ & 117.79 & 118.27 & 0.48 & 6 & OS20 & 0.67 & 1 & $L S A M P, 1 a$ & 1.18 \\
\hline & 117.79 & 118.39 & 0.60 & 7 & OS40 & 0.34 & & & 0.13 \\
\hline & 117.97 & 118.12 & 0.15 & 3 & OS46 & 0.67 & & & 0.12 \\
\hline & 117.79 & 118.39 & 0.60 & 7 & $22 \mathrm{~T}$ & 0.67 & & & 0.46 \\
\hline & 117.79 & 118.39 & 0.60 & 7 & $33 \mathrm{~T}$ & 0.62 & & & 0.08 \\
\hline & 117.97 & 118.20 & 0.23 & 4 & G292 & 0.67 & & & 0.48 \\
\hline & 118.12 & 118.20 & 0.08 & 3 & MG63 & 0.00 & & & 0.00 \\
\hline & 117.79 & 118.54 & 0.75 & 8 & HOS & 0.00 & & & 0.00 \\
\hline $7 q 35$ & 146.12 & 146.46 & 0.34 & 3 & OS40 & 0.34 & 1 & CNTNAP2 & 0.20 \\
\hline & 146.12 & 146.55 & 0.43 & 4 & $33 \mathrm{~T}$ & 0.34 & & & 0.17 \\
\hline & 146.12 & 146.55 & 0.43 & 4 & $\mathrm{U} 2 \mathrm{OS}$ & 0.67 & & & 0.63 \\
\hline $9 p 21.3$ & 21.97 & 22.03 & 0.06 & 3 & OS21 & 0.67 & 2 & $C D K N 2 A$ & 0.42 \\
\hline & 21.97 & 22.21 & 0.24 & 5 & $14 \mathrm{~T}$ & 0.67 & & & 0.31 \\
\hline & 21.97 & 22.21 & 0.24 & 5 & $22 \mathrm{~T}$ & 0.67 & & & 0.62 \\
\hline & 21.97 & 22.21 & 0.24 & 5 & MG63 & 0.00 & & & 0.00 \\
\hline & 21.97 & 22.21 & 0.24 & 5 & HOS & 0.00 & & & 0.00 \\
\hline $9 p 21.3$ & 24.00 & 24.91 & 0.91 & 6 & $14 \mathrm{~T}$ & 0.67 & 1 & LOC389707 & 0.39 \\
\hline & 24.00 & 24.91 & 0.91 & 6 & MG63 & 0.00 & & & 0.03 \\
\hline
\end{tabular}



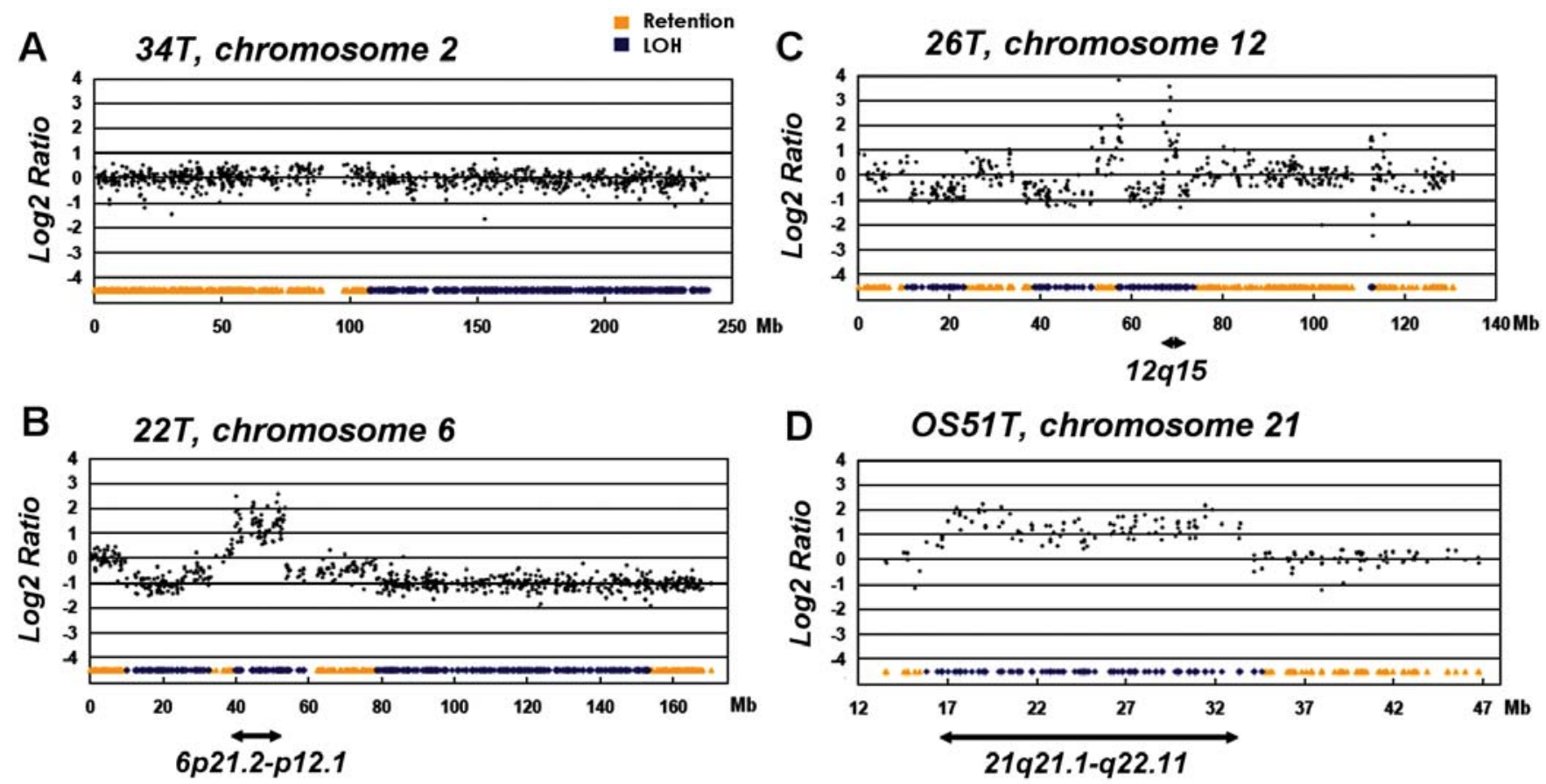

Figure 3. Simultaneous SNP-wise view of copy number $(\mathrm{CN})$ data (log2 ratio, 0 equal two copies) and LOH call (orange, retention; blue, $\mathrm{LOH}$ ) showing LOH with normal CN over chromosome $2 \mathrm{q}$ in case 34T (A); and regions with LOH call associated with increased CN (monoallelic amplifications) over 6p21.2-p12.1 in case 22T (B), 12q15 in case 26T (C) and 21q21.1-q22.11 in case OS51T (D).
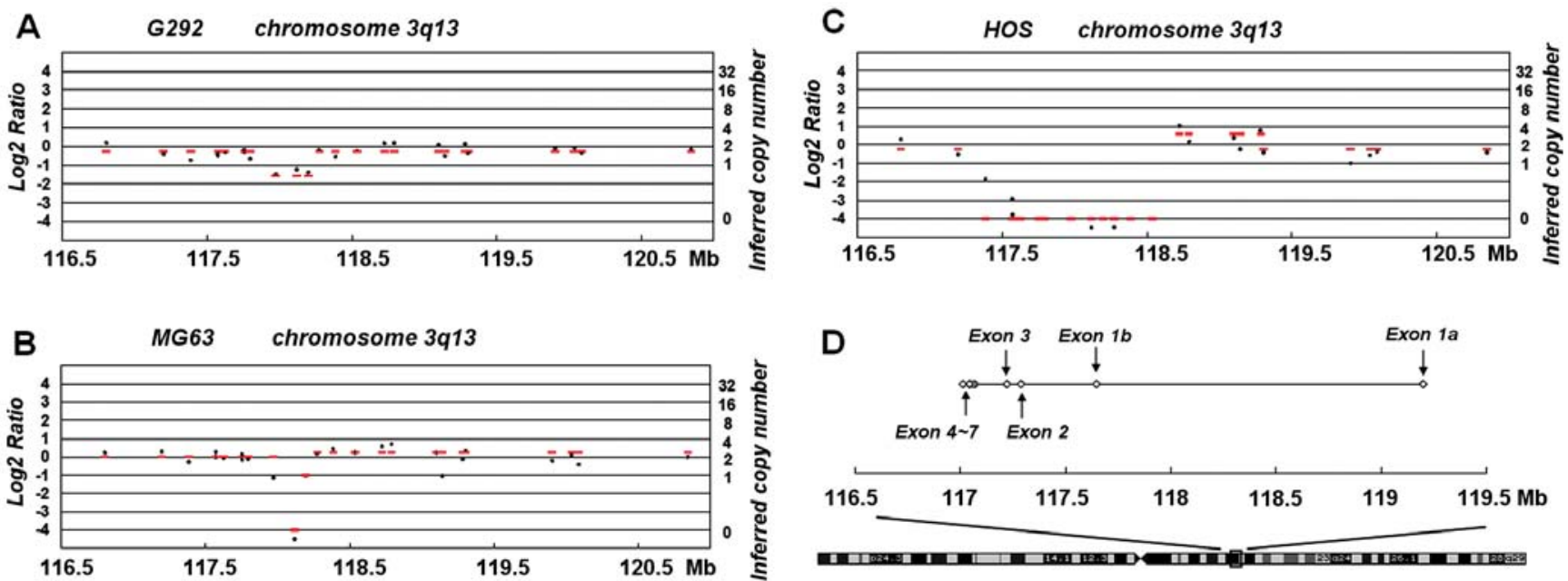

Figure 4. Characterization of 3q13.31 deletion region. Scatter plots of $\log 2$ copy number (CN) ratios (black dots, left axis) and inferred CN (red horizontal bars, right axis) for chromosome band 3q13.31 SNPs showing highly localized deletions in OS cell lines (A) G292 (heterozygous deletion at 117.97-118.20 Mb), (B) MG63 (homozygous deletion at 118.12-118.20 Mb), and (C) HOS (homozygous deletion at 117.39-118.54 Mb). (D) This highly localized deletion lies within the gene encoding limbic system-associated membrane protein (LSAMP), which has two isoforms resulting from use of alternate forms of exon 1. Isoform 1a (exon 1a) spans the genomic region 117.01-119.20 Mb, whereas isoform $1 \mathrm{~b}$ (exon 1b) spans 117.01-117.64 Mb.

However, the 229 bp fragment resulted from the direct splicing of exon 1a to exon 2 , creating a premature stop codon (Fig. 8B-D).

\section{Discussion}

In the present study, we performed a high-resolution genomewide genotyping of OS using SNP array and disclosed a full picture of chromosomal aberrations in OS. We also identified highly amplified regions ranging in size from $32 \mathrm{~Kb}$ to $3.48 \mathrm{Mb}$ and homozygous deletions ranging from 60 to
$910 \mathrm{~Kb}$, which were confirmed by qPCR (Table IV). Among these regions, several (and associated candidate genes) have been reported in previous studies, such as $4 \mathrm{q} 12$ (KIT and PDGFRA) (34,35), 6p21.1 (CDC5L) (36), 12q15 (MDM2) (1), 17p12 (COPS3) (37-39) and 9p21.3 (CDKN2A) (1). However, novel recurrent chromosomal aberrations were also identified, including 7p22.2 (SDK1), 21q21.1 (BTG3) and 3q13.31 (LSAMP).

$\mathrm{CN}$ gains of $8 \mathrm{q}$ and $17 \mathrm{p} 12$ were associated with disease progression. Gain over 8q has been implicated previously in disease progression of OS $(9)$ and other cancers $(40,41)$. In this 
Table V. Chromosomal copy number aberrations associated with progression in OS.

\begin{tabular}{|c|c|c|c|c|c|c|c|}
\hline Cytoband & $\begin{array}{l}\text { Start } \\
(\mathrm{Mb})\end{array}$ & $\begin{array}{l}\text { Stop } \\
(\mathrm{Mb})\end{array}$ & $\begin{array}{l}\text { Size } \\
(\mathrm{Mb})\end{array}$ & $\begin{array}{l}\text { SNP } \\
\text { no. }\end{array}$ & $\begin{array}{c}\text { Primary }(\mathrm{n}=23) \\
\text { no. }(\%)\end{array}$ & $\begin{array}{c}\mathrm{R} / \mathrm{M}(\mathrm{n}=14) \\
\text { no. }(\%)\end{array}$ & P-value \\
\hline \multicolumn{8}{|l|}{$\mathrm{CN}$ amplification } \\
\hline $8 \mathrm{q} 21.12$ & 79.74 & 91.94 & 12.20 & 33 & $0(0.00)$ & $4(28.57)$ & 0.015 \\
\hline $8 \mathrm{q} 22.3-\mathrm{q} 24.3$ & 104.79 & 142.07 & 37.28 & 112 & $2(8.70)$ & $6(42.86)$ & 0.022 \\
\hline $17 \mathrm{p} 12$ & 14.69 & 17.35 & 2.66 & 8 & $3(13.04)$ & $7(50.00)$ & 0.02 \\
\hline $\mathrm{Xp} 11.22$ & 54.34 & 56.77 & 2.43 & 4 & $6(26.09)$ & $0(0.00)$ & 0.043 \\
\hline \multicolumn{8}{|l|}{$\mathrm{CN}$ deletion } \\
\hline $6 q 14.1$ & 83.02 & 85.96 & 2.94 & 14 & $2(8.70)$ & $6(42.86)$ & 0.022 \\
\hline $6 \mathrm{q} 16.2-\mathrm{q} 22.31$ & 99.36 & 121.40 & 22.04 & 80 & $1(4.35)$ & $5(35.71)$ & 0.021 \\
\hline 8p23.2-p12 & 5.26 & 32.65 & 27.39 & 115 & $1(4.35)$ & $5(35.71)$ & 0.021 \\
\hline $13 \mathrm{q} 31.3$ & 92.90 & 114.04 & 21.14 & 90 & $6(26.09)$ & $0(0.00)$ & 0.043 \\
\hline
\end{tabular}

$\mathrm{CN}$, copy number; $\mathrm{R} / \mathrm{M}$, recurrent/metastatic tumor.

Table VI. Regions with LOH call associated with progression in OS.

\begin{tabular}{|c|c|c|c|c|c|c|c|c|}
\hline Cytoband & $\begin{array}{l}\text { Start } \\
(\mathrm{Mb})\end{array}$ & $\begin{array}{l}\text { Stop } \\
(\mathrm{Mb})\end{array}$ & $\begin{array}{l}\text { Size } \\
(\mathrm{Mb})\end{array}$ & $\begin{array}{l}\text { SNP } \\
\text { no. }\end{array}$ & $\begin{array}{c}\text { Primary }(\mathrm{N}=23) \\
\text { no. }(\%)\end{array}$ & $\begin{array}{c}\mathrm{R} / \mathrm{M}(\mathrm{N}=14) \\
\text { no. }(\%)\end{array}$ & P-value & $\begin{array}{c}\text { Reduced } \\
\mathrm{CN}\end{array}$ \\
\hline $2 \mathrm{q} 24.3-\mathrm{q} 31.2$ & 164.56 & 178.77 & 14.21 & 55 & $0(0)$ & $4(28.57)$ & 0.015 & No \\
\hline $5 q 11.2$ & 54.87 & 57.11 & 2.24 & 10 & $0(0)$ & $4(28.57)$ & 0.015 & No \\
\hline $6 \mathrm{p} 21.31-\mathrm{p} 21.1$ & 34.59 & 46.15 & 11.56 & 33 & $1(4.35)$ & $6(42.86)$ & 0.007 & No \\
\hline $6 q 14.1-q 16.2$ & 79.99 & 99.36 & 19.37 & 96 & $4(17.39)$ & $7(50)$ & 0.042 & Yes \\
\hline $8 \mathrm{p} 22-\mathrm{p} 12$ & 13.06 & 32.65 & 19.59 & 94 & $2(8.7)$ & $6(42.86)$ & 0.022 & Yes \\
\hline $9 \mathrm{q} 22.1$ & 88.54 & 97.56 & 9.02 & 26 & $0(0)$ & $4(28.57)$ & 0.015 & No \\
\hline $10 \mathrm{q} 21.1-\mathrm{q} 22.1$ & 57.90 & 72.38 & 14.48 & 65 & $2(8.7)$ & $6(42.86)$ & 0.022 & Yes \\
\hline $10 \mathrm{q} 23.31-\mathrm{q} 24.1$ & 90.41 & 97.48 & 7.07 & 39 & $4(17.39)$ & $7(50)$ & 0.042 & Yes \\
\hline $12 q 15-q 21.1$ & 69.67 & 72.26 & 2.59 & 20 & $1(4.35)$ & $5(35.71)$ & 0.021 & No \\
\hline $21 \mathrm{q} 21.2-\mathrm{q} 21.3$ & 24.36 & 28.29 & 3.93 & 26 & $0(0)$ & $4(28.57)$ & 0.015 & No \\
\hline
\end{tabular}

$\mathrm{CN}$, copy number; R/M, recurrent/metastatic tumor. Reduced $\mathrm{CN}$, indicates whether $>50 \%$ of LOH calls in that region are associated with copy number decrease.

study, we not only confirmed this finding, but also localized the minimal amplification regions to $8 \mathrm{q} 21.12$ and $8 \mathrm{q} 22.3-$ q24.3. These regions are similar to those revealed in studies of disease progression in prostate (40) and gastroesophageal junction adenocarcinoma (41). Amplification of 17p12 was previously identified by OS genotyping (38). COPS3, a candidate oncogene within this region, was amplified and overexpressed in OS (37) and increased $\mathrm{CN}$ of COPS3 was associated with unfavorable prognosis in OS (39). In the present study, recurrent/metastatic tumors were associated with $17 \mathrm{p} 12 \mathrm{CN}$ gain and qPCR confirmed that COPS3 was the most likely candidate within this region (Table IV).

$\mathrm{CN}$ losses at 6q14.1, 6q16.2-q22.31 and 8p23.2-p12 were more frequently demonstrated in recurrent/metastatic OS. Deletion 6q was associated with metastasis in insulinoma (42), and deletions $6 \mathrm{q} 14$ and $6 \mathrm{q} 16-\mathrm{q} 23$ have been reported as recurrent cytogenetic aberrations in OS $(43,44)$. Likewise, previous studies have shown an association of deletion $8 \mathrm{p}$ with disease progression in hepatocellular carcinoma (45) and colon cancer (46). These regions may harbor candidate metastasis suppressors and deserve further studies.

Distinct genetic mechanisms could cause LOH, with different resultant $\mathrm{CN}$ changes (11). Point mutation followed by hemizygous deletion could lead to $\mathrm{CN}$ reductions. These regions often harbor tumor suppressor genes. On the other hand, mutation with subsequent mitotic recombination or gene conversion will not lead to $\mathrm{CN}$ changes (11). CN-neutral LOH has been identified in various human cancers $(13-16,47,48)$, and usually implies tumor suppressor events $(11,47)$. However, sometime it may be associated with activating mutations, such as those identified in myeloid malignancy $(16,49)$.

SNP arrays detected both $\mathrm{CN}$ changes and $\mathrm{LOH}$ and could help in discriminating different underlying mechanisms of $\mathrm{LOH}$ by combining $\mathrm{CN}$ analysis. In this study, we identified chromosomal regions with frequent $\mathrm{LOH}$ at $3 \mathrm{p}, 6 \mathrm{q}, 8 \mathrm{p}, 9 \mathrm{p}, 10 \mathrm{q}$ and $13 \mathrm{q}$, which generally resulted from the classical deletions 

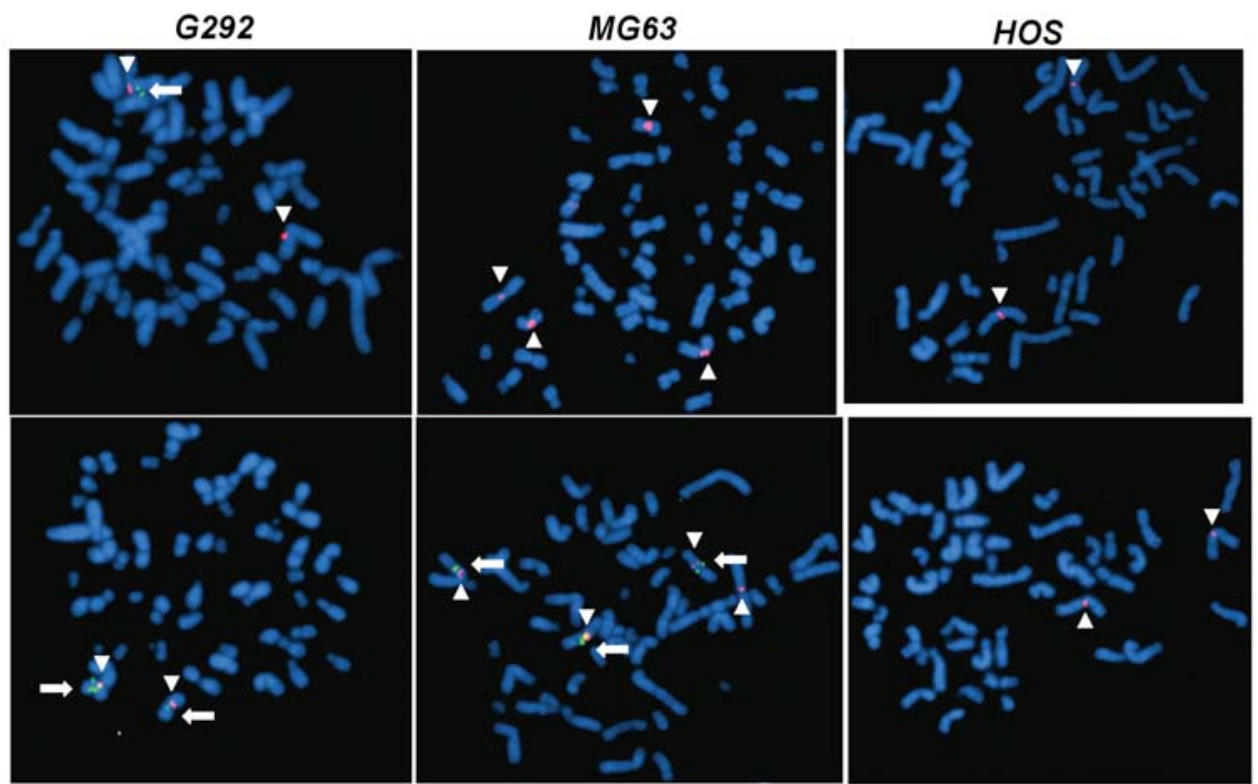

Figure 5. FISH corroboration of 3q13.31 deletions. BAC clones CTD-2119D22 (118.05-118.13 Mb, upper panel) and RP11-33E19 (covering LSAMP exon 1b at 117.46-117.61 Mb, lower panel) were detected with FITC (green, indicated by arrows); whereas chromosome 3 centromere probe was detected with rhodamine (red, indicated by arrowheads). CTD-2119D22 shows heterozygous deletion in G292 and homozygous deletions in MG63 and HOS, whereas RP11-33E19 is deleted only in HOS.
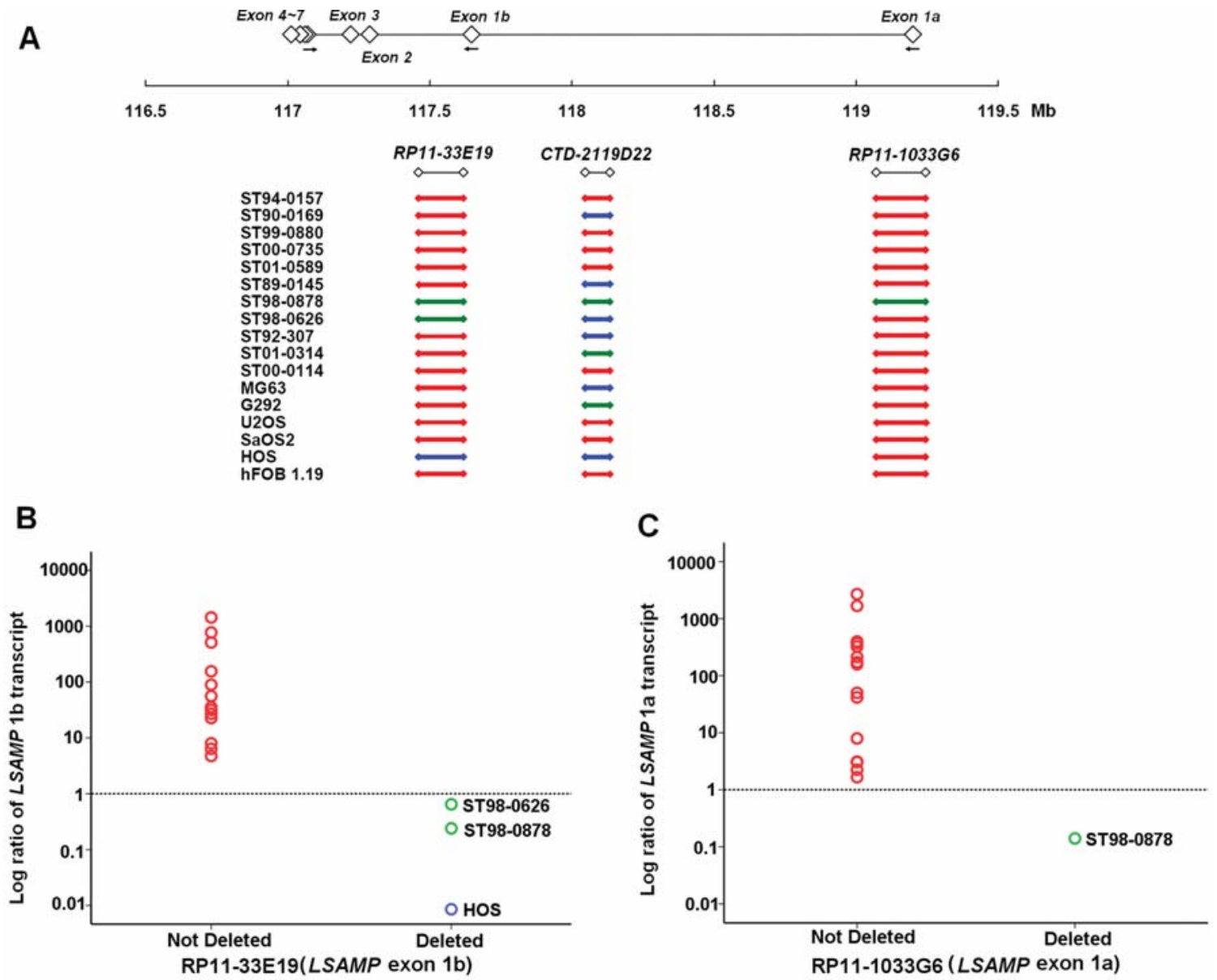

Figure 6. Confirmation of localized LSAMP deletion in primary OSs and correlation of expression with deletion. (A) FISH evaluations are indicated as (blue arrow) homozygous deletion; (green arrow) heterozygous deletion; (red arrow) no deletion. Arrows on the exon schematic show locations of reverse primer in exon 4 and forward primers in exons 1a or 1b, as used in the qRT-PCR studies. Four primary OSs (ST90-0169, ST89-0145, ST92-307, ST01-314) and two OS cell lines (MG63 and G292) had homozygous or heterozygous CTD-2119D22 deletion (118.05-118.13 Mb), between LSAMP exons 1a and 1b. Two primary OSs (ST98-0626 and ST98-0878) and HOS had both CTD-2119D22 and RP11-33E19 deletion (117.46-117.61 Mb, affecting LSAMP exon 1b). ST98-0878 also had RP11-1033G6 deletion (119.07-119.24 Mb, involving LSAMP exon 1a). (B) LSAMP 1b expression, shown in comparison to expression in osteoblast cell line hFOB 1.19 (dashed line), is lowest in OSs with LSAMP exon 1b deletions (ST98-0878, ST98-0626, HOS). (C) LSAMP 1a expression shown in comparison to expression in osteoblast cell line hFOB 1.19 (dashed line), is lowest in OS with LSAMP exon 1a deletion (ST98-0878), 

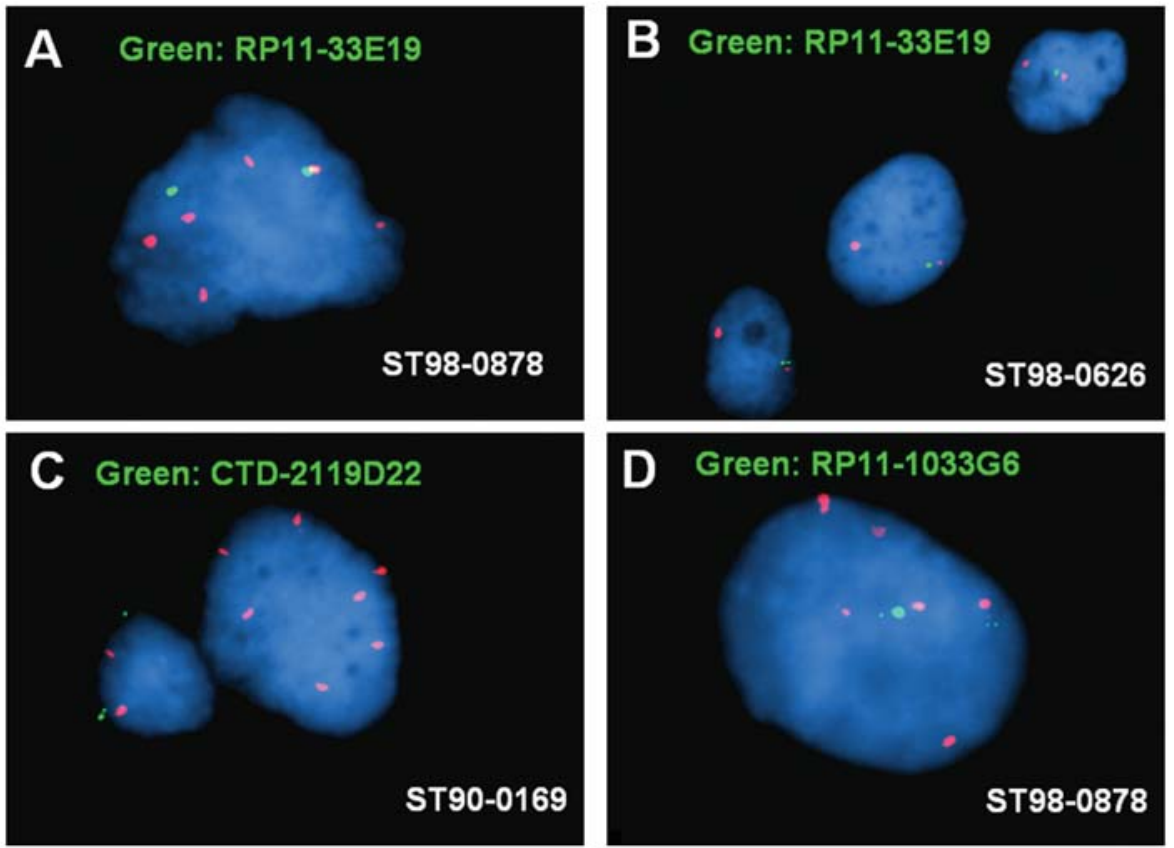

Figure 7. Representative FISH images in frozen OS. Chromosome 3 centromere probe was detected with rhodamine (red). All other BAC probes were detected with FITC (green). (A) Heterozygous deletion of RP11-33E19 (117.46-117.61 Mb, covering LSAMP exon 1b region) in ST98-0878; (B) heterozygous deletion of RP11-33E19 in ST98-0626; (C) homozygous deletion of CTD-2119D22 (118.05-118.13 Mb region) in ST90-0169 with non-neoplastic nucleus for comparison at left; (D) heterozygous deletion of RP11-1033G6 (119.07-119.24 Mb, covering LSAMP exon 1a region) in ST98-0878.

A

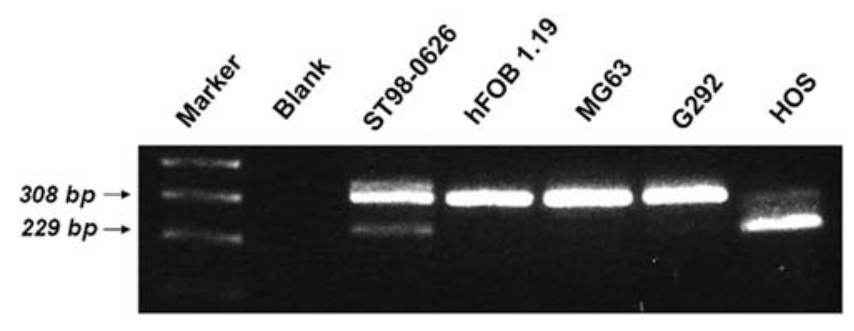

B

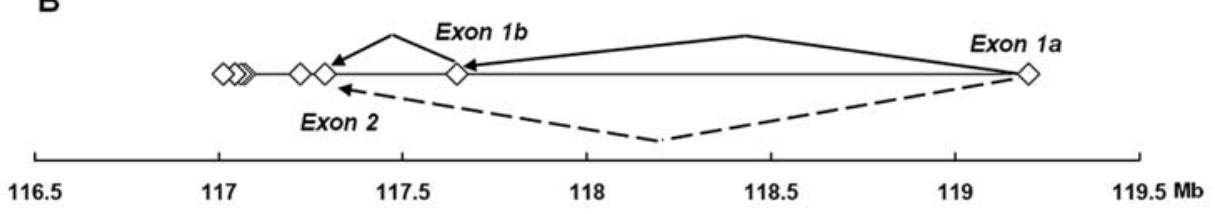

C

Part of the nucleic acid sequence from 308 bp PCR product ATGAGGACCTACTGGCTGCACAGCGTCTGGGTGCTGGGCTTTTTCCTGTCCCTCTTCTCATTGCAAGGACTGCCTGTTCGCAGCGT.Exon 1a

Exon 1b

Corresponding amino acid sequence from 308 bp PCR product MRTYWLHSVWVLGFFLSLFSLQGLPVRS--

\section{D}

Part of the nucleic acid sequence from 229 bp PCR product ATGAGGACCTACTGGCTGCACAGCGTCTGGGTGCTGGGCTTITTCCTGTCCCTCTTCTCATTGCAAGGTGCGTTGTAGAAGACAAG-Exon 1a

Corresponding amino acid sequence from 229 bp PCR product MRTYWLHSVWVLGFFLSLFSLQGAL*KT--

Figure 8. RT-PCR sequencing of LSAMP 1a transcript. (A) An aberrant (229 bp) PCR product is identified in HOS and ST98-0626. (B) The normal 308 bp product consists of $L S A M P$ exon 1 a spliced to the $75^{\text {th }}$ nucleotide of exon $1 \mathrm{~b}$, which is then spliced to exon 2 (solid line and arrow); whereas the aberrant 229 bp product consists of LSAMP exon 1a spliced directly to exon 2 (dashed line and arrow). (C and D) Coding sequences corresponding to the two exon 1a splicing forms. In the $229 \mathrm{bp}$ fragment, a stop codon (arrow) resulted from aberrant splicing. ${ }^{*}$ Position of stop codon. 
with reduced gene dosage. However, we found that $28 \%$ of $\mathrm{LOH}$ events were not associated with $\mathrm{CN}$ alterations. Moreover, we identified regions with $\mathrm{LOH}$ calls associated with increased $\mathrm{CN}$. These regions have been reported in colon cancer (14) and are often associated with allele-specific amplification of oncogenes (17). In our study, it was interesting that the regions with $\mathrm{LOH}$ calls over $12 \mathrm{q} 15$ in case $26 \mathrm{~T}$ contained MDM2, a well-known oncogene in OS (Table IV) (1). We have identified several $\mathrm{CN}$-neutral or $\mathrm{CN}$-increased regions with $\mathrm{LOH}$ calls associated with OS disease progression, which warrant further investigation.

In order to identify chromosomal regions with high-level amplifications and homozygous deletions by SNP array, we adopted the criteria of Zhao et al (19), with modifications. This approach highlighted a novel deletion within the LSAMP gene region over cytoband $3 q 13.31$. LSAMP is a putative tumor suppressor gene, which is downregulated in human renal (50) and ovarian (51) cancers. Silencing LSAMP expression in human aortic smooth muscle cells (SMCs) substantially increased SMCs proliferation (52). LSAMP is a member of the IgLON immunoglobulin subfamily of glycosylphosphatidylinositol-anchored cell adhesion molecules comprised of $L S A M P(53)$, opioid binding protein/cell adhesion moleculelike neurotrimin $(O P C M L)(54,55)$ and neuronal growth regulator 1 (NEGR1) (56). OPCML exhibits functional characteristics of a tumor suppressor gene in ovarian cancer in vitro and in vivo models and is underexpressed in $83 \%$ of sporadic ovarian cancers (57).

Our studies revealed two types of 3q13.31 deletions in OS (Fig. 6A). Type 1 deletions were extremely localized within the 118.05-118.13 Mb region; whereas Type 2 deletions involved a larger region extending from 117.46 to $118.13 \mathrm{Mb}$. Both Type 1 and Type 2 deletions were shown to be somatic in nature, as they were not found in matched non-neoplastic DNAs from several patients. There was no demonstrable correlation between Type 1 deletion and expression levels of $L S A M P 1 \mathrm{a}$ or $1 \mathrm{~b}$ transcripts and therefore the functional relevance of these small deletions remains to be determined. On the other hand, OS cell lines and primary tumors with Type 2 deletions featured low LSAMP $1 \mathrm{~b}$ expression, presumably resulting from deletion of $L S A M P$ exon $1 \mathrm{~b}$. Notably, two OS with Type 2 deletions (ST98-0626 and HOS) featured relatively strong expression of LSAMP 1a transcript. However, RT-PCR and sequencing demonstrated that these LSAMP 1a transcripts were aberrant, because the underlying exon $1 \mathrm{~b}$ deletion engenders splicing of exon 1a directly to exon 2 , creating a shift of the reading frame and a resultant exon 2 premature truncation. In sum, the Type 2 LSAMP deletions are inactivating, being associated with substantial down-regulation of the LSAMP $1 \mathrm{~b}$ transcript and with either reduced expression or presence of a premature termination codon of LSAMP 1a transcripts.

In summary, this study revealed novel chromosomal aberrations in OS and identified cytogenetic changes associated with recurrent/metastatic behavior. The study also demonstrated that $\mathrm{CN}$ and $\mathrm{LOH}$ analyses detect distinct subsets of genetic abnormalities in OS. This genomic profiling in OS revealed a novel highly-localized 3q13.31 deletion and has thereby implicated LSAMP as an OS tumor suppressor.

\section{Acknowledgements}

This study was supported by grants from the Taipei Veterans General Hospital (Grant No. V95C1-123, V96C1-167, V96ER2-005), and National Science Council, Taiwan (Grant No. NSC 93-2314-B-075-029), as well as by Timothy O'Brien Award. This study was also partially supported by the Taiwan Clinical Oncology Research Foundation. The authors acknowledge the technical services support provided by Microarray and Gene Expression Analysis Core Facility of the VGH-Yang Ming Genome Research Center (VYMGC). The Gene Expression Analysis Core Facility is supported by National Research Program for Genomic Medicine, National Science Council, Taiwan, R.O.C. We thank Maureen O'Sullivan for review and helpful comments on the manuscript.

\section{References}

1. Gorlick R, Anderson P, Andrulis I, Arndt C, Beardsley GP, Bernstein M, Bridge J, Cheung NK, Dome JS, Ebb D, Gardner T, Gebhardt M, Grier H, Hansen M, Healey J, Helman L, Hock J, Houghton J, Houghton P, Huvos A, Khanna C, Kieran M, Kleinerman E, Ladanyi M, Lau C, Malkin D, Marina N, Meltzer P, Meyers P, Schofield D, Schwartz C, Smith MA, Toretsky J, Tsokos M, Wexler L, Wigginton J, Withrow S, Schoenfeldt $M$ and Anderson B: Biology of childhood osteogenic sarcoma and potential targets for therapeutic development: meeting summary. Clin Cancer Res 9: 5442-5453, 2003.

2. Khanna C, Wan X, Bose S, Cassaday R, Olomu O, Mendoza A, Yeung C, Gorlick R, Hewitt SM and Helman LJ: The membranecytoskeleton linker ezrin is necessary for osteosarcoma metastasis. Nat Med 10: 182-186, 2004.

3. Bridge JA, Nelson M, McComb E, McGuire MH, Rosenthal H, Vergara G, Maale GE, Spanier S and Neff JR: Cytogenetic findings in 73 osteosarcoma specimens and a review of the literature. Cancer Genet Cytogenet 95: 74-87, 1997.

4. Overholtzer M, Rao PH, Favis R, Lu XY, Elowitz MB, Barany F, Ladanyi M, Gorlick R and Levine AJ: The presence of p53 mutations in human osteosarcomas correlates with high levels of genomic instability. Proc Natl Acad Sci USA 100: 11547-11552, 2003.

5. Squire JA, Pei J, Marrano P, Beheshti B, Bayani J, Lim G, Moldovan L and Zielenska M: High-resolution mapping of amplifications and deletions in pediatric osteosarcoma by use of $\mathrm{CGH}$ analysis of cDNA microarrays. Genes Chromosomes Cancer 38: 215-225, 2003.

6. Stock C, Kager L, Fink FM, Gadner H and Ambros PF: Chromosomal regions involved in the pathogenesis of osteosarcomas. Genes Chromosomes Cancer 28: 329-336, 2000.

7. Man TK, Lu XY, Jaeweon K, Perlaky L, Harris CP, Shah S, Ladanyi M, Gorlick R, Lau CC and Rao PH: Genome-wide array comparative genomic hybridization analysis reveals distinct amplifications in osteosarcoma. BMC Cancer 4: 45, 2004.

8. Atiye J, Wolf M, Kaur S, Monni O, Bohling T, Kivioja A, Tas E, Serra M, Tarkkanen $M$ and Knuutila $S$ : Gene amplifications in osteosarcoma-CGH microarray analysis. Genes Chromosomes Cancer 42: 158-163, 2005.

9. Tarkkanen M, Elomaa I, Blomqvist C, Kivioja AH, Kellokumpu-Lehtinen P, Bohling T, Valle J and Knuutila S: DNA sequence copy number increase at $8 \mathrm{q}$ : a potential new prognostic marker in high-grade osteosarcoma. Int J Cancer 84: 114-121, 1999.

10. Ozaki T, Schaefer KL, Wai D, Buerger H, Flege S, Lindner N, Kevric M, Diallo R, Bankfalvi A, Brinkschmidt C, Juergens H, Winkelmann W, Dockhorn-Dworniczak B, Bielack SS and Poremba C: Genetic imbalances revealed by comparative genomic hybridization in osteosarcomas. Int J Cancer 102: 355-365, 2002.

11. Zhao X, Li C, Paez JG, Chin K, Janne PA, Chen TH, Girard L, Minna J, Christiani D, Leo C, Gray JW, Sellers WR and Meyerson M: An integrated view of copy number and allelic alterations in the cancer genome using single nucleotide polymorphism arrays. Cancer Res 64: 3060-3071, 2004. 
12. Bignell GR, Huang J, Greshock J, Watt S, Butler A, West S, Grigorova M, Jones KW, Wei W, Stratton MR, Futreal PA, Weber B, Shapero MH and Wooster R: High-resolution analysis of DNA copy number using oligonucleotide microarrays. Genome Res 14: 287-295, 2004.

13. Teh MT, Blaydon D, Chaplin T, Foot NJ, Skoulakis S, Raghavan M, Harwood CA, Proby CM, Philpott MP, Young BD and Kelsell DP: Genomewide single nucleotide polymorphism microarray mapping in basal cell carcinomas unveils uniparental disomy as a key somatic event. Cancer Res 65: 8597-8603, 2005.

14. Andersen CL, Wiuf C, Kruhoffer M, Korsgaard M, Laurberg S and Orntoft TF: Frequent occurrence of uniparental disomy in colorectal cancer. Carcinogenesis 28: 38-48, 2007.

15. Raghavan M, Lillington DM, Skoulakis S, Debernardi S, Chaplin T, Foot NJ, Lister TA and Young BD: Genome-wide single nucleotide polymorphism analysis reveals frequent partial uniparental disomy due to somatic recombination in acute myeloid leukemias. Cancer Res 65: 375-378, 2005.

16. Fitzgibbon J, Smith LL, Raghavan M, Smith ML, Debernardi S, Skoulakis S, Lillington D, Lister TA and Young BD: Association between acquired uniparental disomy and homozygous gene mutation in acute myeloid leukemias. Cancer Res 65: 9152-9154, 2005.

17. Wong KK, Tsang YT, Chang YM, Su J, Di Francesco AM, Meco D, Riccardi R, Perlaky L, Dauser RC, Adesina A, Bhattacharjee M, Chintagumpala M and Lau CC: Genome-wide allelic imbalance analysis of pediatric gliomas by single nucleotide polymorphic allele array. Cancer Res 66: 11172-11178, 2006.

18. Gaasenbeek M, Howarth K, Rowan AJ, Gorman PA, Jones A, Chaplin T, Liu Y, Bicknell D, Davison EJ, Fiegler H, Carter NP, Roylance RR and Tomlinson IP: Combined array-comparative genomic hybridization and single-nucleotide polymorphism-loss of heterozygosity analysis reveals complex changes and multiple forms of chromosomal instability in colorectal cancers. Cancer Res 66: 3471-3479, 2006.

19. Zhao X, Weir BA, LaFramboise T, Lin M, Beroukhim R, Garraway L, Beheshti J, Lee JC, Naoki K, Richards WG, Sugarbaker D, Chen F, Rubin MA, Janne PA, Girard L, Minna J, Christiani D, Li C, Sellers WR and Meyerson M: Homozygous deletions and chromosome amplifications in human lung carcinomas revealed by single nucleotide polymorphism array analysis. Cancer Res 65: 5561-5570, 2005

20. Wong KK, Tsang YT, Shen J, Cheng RS, Chang YM, Man TK and Lau CC: Allelic imbalance analysis by high-density singlenucleotide polymorphic allele (SNP) array with whole genome amplified DNA. Nucleic Acids Res 32: e69, 2004.

21. Lin M, Wei LJ, Sellers WR, Lieberfarb M, Wong WH and Li C: dChipSNP: significance curve and clustering of SNP-arraybased loss-of-heterozygosity data. Bioinformatics 20: 1233-1240, 2004.

22. Beroukhim R, Lin M, Park Y, Hao K, Zhao X, Garraway LA, Fox EA, Hochberg EP, Mellinghoff IK, Hofer MD, Descazeaud A, Rubin MA, Meyerson M, Wong WH, Sellers WR and Li C: Inferring loss-of-heterozygosity from unpaired tumors using highdensity oligonucleotide SNP arrays. PLoS Comput Biol 2: e41, 2006.

23. Li C and Wong WH: Model-based analysis of oligonucleotide arrays: model validation, design issues and standard error application. Genome Biol 2: research0032.1-0032.11, 2001.

24. Li C and Wong WH: Model-based analysis of oligonucleotide arrays: expression index computation and outlier detection. Proc Natl Acad Sci USA 98: 31-36, 2001.

25. Yen CC, Chen YJ, Lu KH, Hsia JY, Chen JT, Hu CP, Chen PM, Liu JH, Chiou TJ, Wang WS, Yang MH, Chao TC and Lin CH: Genotypic analysis of esophageal squamous cell carcinoma by molecular cytogenetics and real-time quantitative polymerase chain reaction. Int J Oncol 23: 871-881, 2003.

26. Wang TL, Maierhofer C, Speicher MR, Lengauer C, Vogelstein B, Kinzler KW and Velculescu VE: Digital karyotyping. Proc Natl Acad Sci USA 99: 16156-16161, 2002.

27. Huang SF, Xiao S, Renshaw AA, Loughlin KR, Hudson TJ and Fletcher JA: Fluorescence in situ hybridization evaluation of chromosome deletion patterns in prostate cancer. Am J Pathol 149: 1565-1573, 1996

28. Pinkel D, Landegent J, Collins C, Fuscoe J, Segraves R, Lucas J and Gray J: Fluorescence in situ hybridization with human chromosome-specific libraries: detection of trisomy 21 and translocations of chromosome 4. Proc Natl Acad Sci USA 85: 9138-9142, 1988.
29. Kim UJ, Birren BW, Slepak T, Mancino V, Boysen C, Kang HL, Simon MI and Shizuya H: Construction and characterization of a human bacterial artificial chromosome library. Genomics 34 : 213-218, 1996.

30. Osoegawa K, Mammoser AG, Wu C, Frengen E, Zeng C, Catanese JJ and de Jong PJ: A bacterial artificial chromosome library for sequencing the complete human genome. Genome Res 11: 483-496, 2001 .

31. Pimenta AF and Levitt P: Characterization of the genomic structure of the mouse limbic system-associated membrane protein (Lsamp) gene. Genomics 83: 790-801, 2004.

32. Hui AB, Takano H, Lo KW, Kuo WL, Lam CN, Tong CY, Chang Q, Gray JW and Ng HK: Identification of a novel homozygous deletion region at 6q23.1 in medulloblastomas using high-resolution array comparative genomic hybridization analysis. Clin Cancer Res 11: 4707-4716, 2005.

33. Muller-Tidow C, Metzger R, Kugler K, Diederichs S, Idos G, Thomas M, Dockhorn-Dworniczak B, Schneider PM, Koeffler HP, Berdel WE and Serve H: Cyclin E is the only cyclin-dependent kinase 2-associated cyclin that predicts metastasis and survival in early stage non-small cell lung cancer. Cancer Res 61: 647-653, 2001.

34. Entz-Werle N, Marcellin L, Gaub MP, Guerin E, Schneider A, Berard-Marec P, Kalifa C, Brugiere L, Pacquement H, Schmitt C, Tabone MD, Jeanne-Pasquier C, Terrier P, Dijoud F, Oudet $P$, Lutz $\mathrm{P}$ and Babin-Boilletot A: Prognostic significance of allelic imbalance at the c-kit gene locus and c-kit overexpression by immunohistochemistry in pediatric osteosarcomas. J Clin Oncol 23: 2248-2255, 2005.

35. Entz-Werle N, Gaub MP, Lavaux T, Marcellin L, Metzger N, Marec-Berard P, Schmitt C, Brugiere L, Kalifa C, Tabone MD, Pacquement H, Gentet P, Lutz P, Oudet P and Babin A: KIT gene in pediatric osteosarcomas: could it be a new therapeutic target? Int J Cancer 120: 2510-2516, 2007.

36. Lu XY, Lu Y, Zhao YJ, Jaeweon K, Kang J, Xiao-Nan L, Ge G, Meyer R, Perlaky L, Hicks J, Chintagumpala M, Cai WW, Ladanyi M, Gorlick R, Lau CC, Pati D, Sheldon M and Rao PH: Cell cycle regulator gene CDC5L, a potential target for $6 \mathrm{p} 12-$ p21 amplicon in osteosarcoma. Mol Cancer Res 6: 937-946, 2008.

37. Henriksen J, Aagesen TH, Maelandsmo GM, Lothe RA, Myklebost $\mathrm{O}$ and Forus A: Amplification and overexpression of COPS3 in osteosarcomas potentially target TP53 for proteasomemediated degradation. Oncogene 22: 5358-5361, 2003.

38. van DM and Hulsebos TJ: Amplification and overexpression of genes in $17 \mathrm{p} 11.2 \sim$ p12 in osteosarcoma. Cancer Genet Cytogenet 153: 77-80, 2004

39. Yan T, Wunder JS, Gokgoz N, Gill M, Eskandarian S, Parkes RK, Bull SB, Bell RS and Andrulis IL: COPS3 amplification and clinical outcome in osteosarcoma. Cancer 109: 1870-1876, 2007.

40. van DM, van MR, Vissers K, Watson JE, van Weerden WM, Schroder FH, Hop WC, van der Kwast TH, Collins C and van DH: High-resolution array comparative genomic hybridization of chromosome arm 8q: evaluation of genetic progression markers for prostate cancer. Genes Chromosomes Cancer 44: 438-449, 2005.

41. van DM, van MR, Vissers KJ, Hop WC, Dinjens WN, Tilanus HW, Siersema PD and van DH: High-resolution array comparative genomic hybridization of chromosome 8q: evaluation of putative progression markers for gastroesophageal junction adenocarcinomas. Cytogenet Genome Res 118: 130-137, 2007.

42. Jonkers YM, Claessen SM, Perren A, Schmid S, Komminoth P, Verhofstad AA, Hofland LJ, de Krijger RR, Slootweg PJ, Ramaekers FC and Speel EJ: Chromosomal instability predicts metastatic disease in patients with insulinomas. Endocr Relat Cancer 12: 435-447, 2005.

43. Nathrath MH, Kuosaite V, Rosemann M, Kremer M, Poremba C, Wakana S, Yanagi M, Nathrath WB, Hofler H, Imai K and Atkinson MJ: Two novel tumor suppressor gene loci on chromosome $6 \mathrm{q}$ and $15 \mathrm{q}$ in human osteosarcoma identified through comparative study of allelic imbalances in mouse and man. Oncogene 21: 5975-5980, 2002

44. Ohata N, Ito S, Yoshida A, Kunisada T, Numoto K, Jitsumori Y, Kanzaki H, Ozaki T, Shimizu K and Ouchida M: Highly frequent allelic loss of chromosome 6q16-23 in osteosarcoma: involvement of cyclin C in osteosarcoma. Int J Mol Med 18: 1153-1158, 2006.

45. Lu T and Hano H: Identification of minimal regions of deletion at 8 p23.1-22 associated with metastasis of hepatocellular carcinoma. Liver Int 27: 782-790, 2007. 
46. Knosel T, Schluns K, Dietel M and Petersen I: Chromosomal alterations in lung metastases of colorectal carcinomas: associations with tissue specific tumor dissemination. Clin Exp Metastasis 22: 533-538, 2005.

47. Murthy SK, DiFrancesco LM, Ogilvie RT and Demetrick DJ: Loss of heterozygosity associated with uniparental disomy in breast carcinoma. Mod Pathol 15: 1241-1250, 2002.

48. White VA, McNeil BK and Horsman DE: Acquired homozygosity (isodisomy) of chromosome 3 in uveal melanoma. Cancer Genet Cytogenet 102: 40-45, 1998.

49. Jones AV, Kreil S, Zoi K, Waghorn K, Curtis C, Zhang L, Score J, Seear R, Chase AJ, Grand FH, White H, Zoi C, Loukopoulos D, Terpos E, Vervessou EC, Schultheis B, Emig M, Ernst T, Lengfelder E, Hehlmann R, Hochhaus A, Oscier D, Silver RT, Reiter A and Cross NC: Widespread occurrence of the JAK2 V617F mutation in chronic myeloproliferative disorders. Blood 106: 2162-2168, 2005

50. Chen J, Lui WO, Vos MD, Clark GJ, Takahashi M, Schoumans J, Khoo SK, Petillo D, Lavery T, Sugimura J, Astuti D, Zhang C, Kagawa S, Maher ER, Larsson C, Alberts AS, Kanayama HO and Teh BT: The t $(1 ; 3)$ breakpoint-spanning genes LSAMP and NORE1 are involved in clear cell renal cell carcinomas. Cancer Cell 4: 405-413, 2003.

51. Ntougkos E, Rush R, Scott D, Frankenberg T, Gabra H, Smyth JF and Sellar GC: The IgLON family in epithelial ovarian cancer: expression profiles and clinicopathologic correlates. Clin Cancer Res 11: 5764-5768, 2005.

52. Wang L, Hauser ER, Shah SH, Seo D, Sivashanmugam P, Exum ST, Gregory SG, Granger CB, Haines JL, Jones CJ, Crossman D, Haynes C, Kraus WE, Freedman NJ, PericakVance MA, Goldschmidt-Clermont PJ and Vance JM: Polymorphisms of the tumor suppressor gene LSAMP are associated with left main coronary artery disease. Ann Hum Genet 72: 443-453, 2008.
53. Levitt P: A monoclonal antibody to limbic system neurons. Science 223: 299-301, 1984.

54. Struyk AF, Canoll PD, Wolfgang MJ, Rosen CL, D'Eustachio P and Salzer JL: Cloning of neurotrimin defines a new subfamily of differentially expressed neural cell adhesion molecules. J Neurosci 15: 2141-2156, 1995.

55. Schofield PR, McFarland KC, Hayflick JS, Wilcox JN, Cho TM, Roy S, Lee NM, Loh HH and Seeburg PH: Molecular characterization of a new immunoglobulin superfamily protein with potential roles in opioid binding and cell contact. EMBO J 8: 489-495, 1989.

56. Funatsu N, Miyata S, Kumanogoh H, Shigeta M, Hamada K, Endo Y, Sokawa Y and Maekawa S: Characterization of a novel rat brain glycosylphosphatidylinositol-anchored protein (Kilon), a member of the IgLON cell adhesion molecule family. J Biol Chem 274: 8224-8230, 1999.

57. Sellar GC, Watt KP, Rabiasz GJ, Stronach EA, Li L, Miller EP, Massie CE, Miller J, Contreras-Moreira B, Scott D, Brown I, Williams AR, Bates PA, Smyth JF and Gabra H: OPCML at $11 \mathrm{q} 25$ is epigenetically inactivated and has tumor-suppressor function in epithelial ovarian cancer. Nat Genet 34: 337-343, 2003. 